\title{
Certificate-Based Encryption Resilient to Key Leakage
}

\author{
Qihong Yua ${ }^{\mathrm{a}}$, Jiguo $\mathrm{Li}^{\mathrm{a}}{ }^{1}$, Yichen Zhang ${ }^{\mathrm{a}}$, Wei Wu ${ }^{\mathrm{b}, \mathrm{c}}$, Xinyi Huang ${ }^{\mathrm{b}, \mathrm{c}}$, Yang Xiang ${ }^{\mathrm{c}}$ \\ ${ }^{a}$ College of Computer and Information, Hohai University, Nanjing 211100, China \\ ${ }^{\mathrm{b}}$ School of Mathematics and Computer Science, Fujian Normal University, Fuzhou, China, 350117 \\ ${ }^{\mathrm{c}}$ School of Information Technology, Deakin University, Burwood, VIC 3125, Australia
}

\begin{abstract}
Certificate-based encryption (CBE) is an important class of public key encryption but the existing schemes are secure only under the premise that the decryption key (or private key) and master private key are absolutely secret. In fact, a lot of side channel attacks and cold boot attacks can leak secret information of a cryptographic system. In this case, the security of the cryptographic system is destroyed, so a new model called leakage-resilient (LR) cryptography is introduced to solve this problem. While some traditional public key encryption and identity-based encryption with resilient-leakage schemes have been constructed, as far as we know, there is no leakage-resilient scheme in certificate-based cryptosystems. This paper puts forward the first certificate-based encryption scheme which can resist not only the decryption key leakage but also the master secret key leakage. Based on composite order bilinear group assumption, the security of the scheme is proved by using dual system encryption. The relative leakage rate of key is close to $1 / 3$.
\end{abstract}

Keywords: certificate-based encryption, master secret key leakage, dual system encryption, composite order bilinear group

\section{Introduction}

In order to solve certificate management problem in traditional public key cryptosystems and the key escrow problem in identity based cryptosystems, Gentry [1] proposed a new cryptography paradigm called certificate-based encryption. From then on, many concrete schemes $[2,3,4,5,6,7,8]$ were constructed under the assumption that the decryption key and master secret key are absolutely confidential.

But that is not always the case, and some side channel attacks $[9,10,11,12,13]$ have been found in real world. From the attacks, the adversary can obtain some information by observing execution timing, energy consumption, etc. This results in secret information leakage which includes the information of the vital master secret key and decryption key. Side channel attacks give the adversaries an advantage to obtain the secret information. Therefore, the security of previous cryptographic schemes is compromised under the circumstances. New model must be constructed to capture such attacks.

In order to guarantee the security of cryptographic systems under some circumstances, we usually define an attack model to limit the attacker's behavior. If the attacker satisfies the constraints, the corresponding cryptosystems are regarded as safe in the model. Leakage resilient cryptography is to capture side channel attacks. In fact, it has become a research hotspot in recent years.

For identity-based cryptosystems and traditional public key cryptosystems, some leakage-resilient schemes have been constructed. For certificate-based cryptosystems, as far as we know, no leakage-resilient scheme is presented. The paper puts forward the first certificate-based encryption scheme resilient to master secret key leakage and decryption key leakage.

\subsection{Related Work}

In 2004, Micali and Reyzin [14] proposed "only computation leaks information" model: computation is divided into many steps. Only the part of the secret state which is accessed (i.e. active) in that step can leak. The other part of the secret state that is not accessed (i.e. inactive) will not leak in that step. Under this model, the leakage-resilient stream cipher $[15,16]$ and leakage-resilient signature [17] were constructed. Although "only computation leaks information" model describes a large class of leakage attacks, it has shortcomings, namely, it does not capture the setting where the inactive part in memory also leaks information (for example, the cold boot attack [9]). In order to solve this problem, the work [18] introduced "bounded leakage" model, it is a stronger model than "only computation leaks information" model. In "bounded leakage" model, the leakage of inactive part is also considered. Under the "bounded leakage" model, leakage-resilient encryption and signature schemes [19, 20,21] were

1 The corresponding author: Email: ljg1688@163.com, lijiguo@hhu.edu.cn 
constructed. The constructions of leakage-resilient identity-based schemes attract more attention. Some achievements have been given in the works [22, 23, 24].

By constructing the hash proof system, the work [20] gave the leakage-resilient encryption scheme which can resist $l / 4$ bits information leakage about private key ( $l$ is the bit length of private key). The work [25] extended the method of the work [20] to construct the identity-based hash proof system and further to put forward the leakage-resilient identity-based encryption (LR-IBE) in the bounded retrieval model. To improve the property of leakage resilience, the work [26] introduced the dual system encryption.

\subsection{Our Contribution}

Similar to traditional security model of CBE, we consider two types of adversaries as well. The first type of adversary $\mathcal{A}_{1}$ is the malicious user who is allowed to replace public key without knowing the master secret key. The second type of adversary $\mathcal{A}_{2}$ is the dishonest certificate authority (CA) who has the master secret key for generating the certificate but he is not allowed to replace the public key. Inspired by the leakage-resilient certificateless encryption (CLE) [27] and the certificate-based encryption [28], we propose the formal definition and the security model of the leakage-resilient certificate-based encryption (LR-CBE) and further present the first leakage-resilient certificate-based encryption scheme in the "bounded leakage" model. The security of the scheme has been proved by utilizing dual system encryption technique. The leakage bound amounts to $1 / 3$ if $n$ is large enough. Performance comparison illustrates the encryption operation of our scheme is faster than that of the schemes given in [1]. However, decryption cost is linearly correlated with the vector size $n$. In order to make the scheme more efficient, we can take $n=2$ and the decryption operation needs 4 pairings which is acceptable in practical application.

\subsection{Our Technique}

In the security proof we use dual system encryption technique proposed in [29]. The dual system encryption technique can be used to improve the security of cryptographic systems. In the dual system encryption the decryption keys and ciphertexts have two states: normal and semi-functional (SF). The normal decryption keys can decrypt the normal and semi-functional ciphertexts. The semi-functional decryption keys can only decrypt the normal ciphertexts correctly. In real security game, all decryption keys and ciphertexts are normal. The security proof is a hybrid argument where the ciphertexts are first altered to semi-functional ones, then, the keys are altered to semi-functional ones gradually. For the consecutive two games we prove that the attacker cannot detect the difference between them with non-negligible advantage. Finally, we give such a game: we only need to produce semi-functional decryption keys and ciphertexts. Thus the attacker cannot correctly decrypt. This allows us to prove security.

\subsection{Organization}

In Section 2, we give some preliminaries that will be used. Formal description and security model of LR-CBE are given in Section 3. In Section 4, concrete construction of LR-CBE is put forward. Security proof of the proposed scheme is shown in Section 5. The leakage bound is analyzed in Section 6. The comparisons with other schemes are given in Section 7. Section 8 concludes this paper.

\section{Preliminaries}

\subsection{Several Basic Conceptions}

\section{Definition 1: Bilinear Map}

Let $G$ and $G_{T}$ be multiplicative cyclic groups of order $q$ and $P$ be a generator of $G$, a bilinear map $e: G \times G \rightarrow G_{T}$ has three properties as follows:

(1) Bilinearity: For $P, Q \in G$ and $a, b \in Z^{*}, e\left(P^{a}, Q^{b}\right)=e(P, Q)^{a b}$.

(2) Non-degeneracy: For $P, Q \in G, e(P, Q) \neq 1$.

(3) Computability: There is an effective algorithm to calculate $e(P, Q) \in G_{T}$.

Definition 2: NIZK Proof System 
Let $R$ be a binary relation in a language $L$. For $(x, w) \in R, x$ is called the statement and $w$ is called the witness. A non-interactive zero-knowledge (NIZK) proof system consists of three algorithms (Gen, Prf, Ver). The algorithm Gen takes as input a security parameter $1^{\vartheta}$ and outputs the common reference string $\operatorname{crs}$. The prover $\operatorname{Prf}$ takes as input $(\operatorname{crs}, x, w)$ and gives an argument or proof $\pi$ if $(x, w) \in R$. The verifier $\operatorname{Ver}$ takes as input $(\operatorname{crs}, x, \pi)$ and outputs "accept" or "reject". We call (Gen, Prf, Ver) an NIZK proof system for the relation $R$ if it has three properties: soundness, completeness and zero knowledge as [31].

\section{Definition 3: Collision-Resistant Hash Function}

For the hash function $\bar{H}:\{0,1\}^{*} \rightarrow\{0,1\}^{k}$, the algorithm $A$ can obtain the advantage $\varepsilon$ in breaking the collision-resistance of $\bar{H}$ if $\operatorname{Pr}\left[A(\bar{H})=\left(m_{0}, m_{1}\right): m_{0} \neq m_{1}, \bar{H}\left(m_{0}\right)=\bar{H}\left(m_{1}\right)\right] \geq \varepsilon$, where the advantage is over the random bits of $A$. A hash function is collision-resistant if the advantage that any probabilistic polynomial-time (PPT) adversary can obtain is negligible.

\subsection{Complexity Assumptions}

\subsubsection{Composite Order Bilinear Groups}

Composite order bilinear groups are first introduced in [32]. Let $\psi$ denote a generator algorithm of composite order bilinear groups. $\psi$ takes as input a security parameter and outputs a description of composite order bilinear groups $\Omega=\left\{N=p_{1} p_{2} p_{3}, G, G_{T}, e\right\}$, where $p_{1}, p_{2}, p_{3}$ are three $\lambda$-bit primes (The $\lambda$ is related to the security parameter and has an influence on the leakage bound which will be analyzed in Section 6), $G$ and $G_{T}$ are cyclic groups of order $N=p_{1} p_{2} p_{3}$ and $e$ is a bilinear map: $G \times G \rightarrow G_{T}$.

Denote $G_{p_{1}}, G_{p_{2}}$ and $G_{p_{3}}$ as the subgroups of $G$ with order $p_{1}, p_{2}, p_{3}$ respectively. If $h_{i} \in G_{p_{i}}, h_{j} \in G_{p_{j}}$ and $i \neq j$, we have $e\left(h_{i}, h_{j}\right)=1$. For example, suppose $h_{1} \in G_{p_{1}}, h_{2} \in G_{p_{2}}$, and $g$ is a generator of $G$. Thus, $g^{p_{1} p_{2}}$ is a generator of $G_{p_{3}}, g^{p_{1} p_{3}}$ is a generator of $G_{p_{2}}$ and $g^{p_{2} p_{3}}$ is a generator of $G_{p_{1}}$. So, there exists $\alpha_{1}, \alpha_{2}$ such that $h_{1}=\left(g^{p_{2} p_{3}}\right)^{\alpha_{1}}$ and $h_{2}=\left(g^{p_{1} p_{3}}\right)^{\alpha_{2}}$. Then, $e\left(h_{1}, h_{2}\right)=e\left(g^{p_{2} p_{3} \alpha_{1}}, g^{p_{1} p_{3} \alpha_{2}}\right)=e\left(g^{\alpha_{1}}, g^{p_{3} \alpha_{2}}\right)^{p_{1} p_{2} p_{3}}=1$. Therefore, $G_{p_{1}}, G_{p_{2}}$ and $G_{p_{3}}$ are mutual orthogonal.

If an element $X$ can be written uniquely as the product of an element of $G_{p_{1}}$ and an element of $G_{p_{2}}$, we call them " $G_{p_{1}}$ part of $X$ " and " $G_{p_{2}}$ part of $X$ " respectively.

We denote vectors by angle brackets $\langle\cdot, \cdot, \cdot\rangle$ and denote collections of elements of different types by parentheses $(\cdot,,, \cdot)$. Denote dot product of vectors by $\cdot$ and denote component-wise multiplication by $*$. We denote the size or number of bits of the term $W$ as $|W|$.

We define the exponentiation for vectors as follows: For $g \in G, \vec{u}=<u_{1}, u_{2}, \ldots, u_{n}>\in G^{n}$, $a \in Z_{N}, \vec{b}=<b_{1}, b_{2}, \ldots, b_{n}>\in Z_{N}$, we define $g^{\vec{b}}=<g^{b_{1}}, g^{b_{2}}, \ldots, g^{b_{n}}>, \vec{u}^{a}=<u_{1}{ }^{a}, u_{2}{ }^{a}, \ldots, u_{n}{ }^{a}>$. The resulting terms are elements of $G^{n}$. For a bilinear group $G$, we define the pairing operation in $G^{n}$ : For $\vec{u}=<u_{1}, u_{2}, \ldots, u_{n}>\in G^{n}$ and $\vec{v}=<v_{1}, v_{2}, \ldots, v_{n}>\in G^{n}$, the pairing is $e(\vec{u}, \vec{v})$ $=\prod_{i=1}^{n} e\left(u_{i}, v_{i}\right) \in G_{T}$.

\subsubsection{Three Assumptions}

Here we review three assumptions given in $[27,29,30]$ which will be used in our security proof. Denote $G_{p_{1} p_{2}}$ as the subgroup of $G$ with order $p_{1} p_{2}$. 
Let $\psi$ be a generator algorithm of composite order bilinear groups. On input a security parameter $1^{\vartheta}, \psi$ outputs a description of composite order bilinear groups. That is, $\left(N, G, G_{T}, e\right) \stackrel{R}{\longleftarrow} \psi$, where $N=p_{1} p_{2} p_{3}$. Let $g_{1}, g_{2}$ and $g_{3}$ be the generators of $G_{p_{1}}, G_{p_{2}}$ and $G_{p_{3}}$, respectively.

Assumption 1: Given $D^{1}=\left(N, G, G_{T}, e, g_{1}, g_{3}\right)$, no PPT adversary succeeds in distinguishing $T_{0}^{1}=g_{1}^{z}$ from $T_{1}^{1}=g_{1}^{z} g_{2}^{v}$ with non-negligible advantage, where $z, v \in Z_{N}$.

The advantage that adversary $\mathcal{A}$ breaks assumption 1 is defined as:

$$
A d 1_{\psi, \mathcal{A}}(\vartheta)=\left|\operatorname{Pr}\left[\mathcal{A}\left(D^{1}, T_{0}^{1}\right)=1\right]-\operatorname{Pr}\left[\mathcal{A}\left(D^{1}, T_{1}^{1}\right)=1\right]\right| .
$$

We say that assumption 1 holds if the advantage $A d v 1_{\psi, \mathcal{A}}(\vartheta)$ is negligible for any PPT adversary.

Assumption 2: Given $D^{2}=\left(N, G, G_{T}, e, g_{1}, g_{3}, g_{1}^{z} g_{2}^{v}, g_{2}^{u} g_{3}^{\rho}\right)$ where $z, v, u, \rho \in Z_{N}$, no PPT adversary succeeds in distinguishing $T_{0}^{2}=g_{1}^{\omega} g_{3}^{\sigma}$ from $T_{1}^{2}=g_{1}^{\omega} g_{2}^{\kappa} g_{3}^{\sigma}$ with non-negligible advantage, where $\omega, \kappa, \sigma \in Z_{N}$.

The advantage that adversary $\mathcal{A}$ breaks assumption 2 is defined as:

$$
A d v 2_{\psi, \mathcal{A}}(\vartheta)=\left|\operatorname{Pr}\left[\mathcal{A}\left(D^{2}, T_{0}^{2}\right)=1\right]-\operatorname{Pr}\left[\mathcal{A}\left(D^{2}, T_{1}^{2}\right)=1\right]\right| .
$$

We say that assumption 2 holds if the advantage $A d v 2_{\psi, \mathcal{A}}(\vartheta)$ is negligible for any PPT adversary.

Assumption 3: Given $D^{3}=\left(N, G, G_{T}, e, g_{1}, g_{2}, g_{3}, g_{1}^{\alpha} g_{2}^{v}, g_{1}^{s} g_{2}^{u}\right)$ where $\alpha, \mathrm{s}, v, u \in Z_{N}$, no PPT adversary succeeds in distinguishing $T_{0}^{3}=g_{1}^{\alpha z}$ from $T_{1}^{3} \in G_{p_{1}}$ with non-negligible advantage.

The advantage that adversary $\mathcal{A}$ breaks assumption 3 is defined as:

$$
A d 3_{\psi, \mathcal{A}}(\vartheta)=\left|\operatorname{Pr}\left[\mathcal{A}\left(D^{3}, T_{0}^{3}\right)=1\right]-\operatorname{Pr}\left[\mathcal{A}\left(D^{3}, T_{1}^{3}\right)=1\right]\right| .
$$

We say that assumption 3 holds if the advantage $A d v 3_{\psi, \mathcal{A}}(\vartheta)$ is negligible for any PPT adversary.

\section{Formal Definition and Security Model of LR-CBE}

\subsection{Formal Definition of LR-CBE}

Inspired by the works [26, 27], we put forward the formal definition of LR-CBE which is resilient to master secret key leakage and decryption key leakage. We will use a hash function: $\bar{H}: \mathcal{I D} \times \mathcal{P K} \rightarrow \mathcal{I D}$, where $\mathcal{I D}$ is the identity space and $\mathcal{P K}$ is the public key space. The functionality of the hash function is to maintain the security when a CLE is converted to a CBE ( Please refer to [28]). Our LR-CBE scheme is composed of the following seven algorithms.

Setup: $\operatorname{Setup}\left(1^{\vartheta}\right) \rightarrow(m p k, m s k)$. The algorithm is run by the CA. By taking a security parameter $1^{\vartheta}$ as input, the algorithm generates the master public key $m p k$ and the master secret key $m s k$. The $m p k$ is public to all users. The $m p k$ includes the information presentation of the identity space.

SetPrivateKey: SetPrivateKey $(I D, m p k) \rightarrow s k_{I D}$. The algorithm is run by the user. It takes as input the master public key $m p k$ and the identity $I D$. It outputs the user's private key $s k_{I D}$.

SetPublicKey: SetPublicKey $\left(I D, s k_{I D}, m p k\right) \rightarrow p k_{I D}$. The algorithm is run by the user ID. It takes as input the master public key $m p k$ and the private key $s k_{I D}$. It outputs the user's public key $p k_{I D}$.

SetCertificate: SetCertificate $\left(I D, p k_{I D}, m p k, m s k\right) \rightarrow \operatorname{Cert}_{I D}$. The algorithm is run by CA. For an identity $I D$, it first calculates $\bar{H}\left(I D, p k_{I D}\right) \rightarrow I D^{\prime}$. Then, it takes as input the master public key $m p k$, the master secret key $m s k, I D^{\prime}$ and the public $p k_{I D}$. It outputs the user's certificate Cert $_{I D}$.

Encrypt: Encrypt $\left(I D, m p k, M, p k_{I D}\right) \rightarrow C$. The algorithm is run by the sender. It takes as input 
the master public key $m p k$, the plaintext $M$, the receiver's identity $I D$ and its corresponding public key $p k_{I D}$. It outputs the ciphertext $C$.

SetDecryptKey: SetDecryptKey $\left(s k_{I D}, \operatorname{Cert}_{I D}\right) \rightarrow d k_{I D}$. The algorithm generates the decryption key $d k_{I D}$ by using $s k_{I D}$ and $\operatorname{Cert}_{I D}$.

Decrypt: $\operatorname{Decrypt}\left(m p k, C, d k_{I D}\right) \rightarrow M$. The algorithm is run by the receiver. It takes as input the master public key $m p k$, the ciphertext $C$, the decryption key $d k_{I D}$ which is generated by using $s k_{I D}$ and $\operatorname{Cert}_{I D}$. It outputs the plaintext $M$.

\subsection{Adversaries and Oracles of LR-CBE}

We consider two types of adversaries in our model like the works [2-8]. One type of adversaries is denoted by $\mathcal{A}_{1}$. Another type of adversaries is denoted by $\mathcal{A}_{2} . \mathcal{A}_{1}$ acts as the dishonest users. $\mathcal{A}_{1}$ can not query the certificate of the target user but he is allowed to replace any user's public key. $\mathcal{A}_{2}$ acts as the CA. $\mathcal{A}_{2}$ can not replace the public key of the target user but he may generate any user's certificate.

The security of LR-CBE against $\mathcal{A} 1$ is defined by the game $\mathcal{T}_{1}$ Game $R$ which will be given in the next subsection. In the game the challenger holds a list: $\mathcal{L}_{1}=\left(H, I D, I D^{\prime}, p k_{I D}, s k_{I D}, C_{e r t}\right.$, $\left.d k_{I D}, l_{d k}, l_{m s k}\right)$. The item consists of a handle counter, an identity, a hashed identity, a public key, a private key, a certificate, a decryption key, the amount of decryption key leakage and the amount of master secret key leakage. When an attacker makes a create query $\mathcal{O}$-Create (Please refer to the concrete definition in the following), the challenger generates a unique handle $H$ and a related item $\left(H, I D, I D^{\prime}, p k_{I D}, s k_{I D}, \perp, \perp, 0,0\right)$. Given a handle, an adversary can make leakage query. After the leakage query, the value of $l_{d k}$ or $l_{m s k}$ which is initially zero will be updated. The other oracle queries will refer the handle.

The security of LR-CBE against $\mathcal{A}_{2}$ is defined by the game $\mathcal{T}_{2}$ _Game_ $R$ which will be given in the next subsection. In the game the challenger holds a list: $\mathcal{L}_{2}=\left(H, I D, I D^{\prime}, p k_{I D}, s k_{I D}, \operatorname{Cert}_{I D}\right.$, $\left.d k_{I D}, l_{d k}\right)$. The item consists of a handle counter, an identity, a hashed identity, a public key, a private key, a certificate, a decryption key and the amount of decryption key leakage. When an attacker makes a create query, the challenger generates a unique handle $H$ and a related item $\left(H, I D, I D^{\prime}, p k_{I D}\right.$, $\left.s k_{I D}, \perp, \perp, 0\right)$. Given a handle, an adversary can make leakage query. After the leakage query, the value of $l_{d k}$ which is initially zero will be updated. The other oracle queries will refer the handle.

We give the oracles that will be used.

(1) $\mathcal{O}$-Create : On the given identity $I D$ from the adversary, the challenger $\mathcal{C}$ does as follows. $\operatorname{SetPrivateKey}(I D, m p k) \rightarrow s k_{I D}, \operatorname{SetPublicKey}\left(I D, s k_{I D}, m p k\right) \rightarrow p k_{I D}$. For the adversary $\mathcal{A} 1$, it adds an item $\left(H, I D, I D^{\prime}, p k_{I D}, s k_{I D}, \perp, \perp, 0,0\right)$ in $\mathcal{L}_{1}$. For adversary $\mathcal{A}_{2}$, it calculates: $I D^{\prime} \leftarrow \bar{H}\left(I D, p k_{I D}\right)$, SetCertificate $\left(I D^{\prime}, p k_{I D}, m p k, m s k\right) \rightarrow \operatorname{Cert}_{I D}$. It adds an item $(H, I D$, $\left.I D^{\prime}, p k_{I D}, s k_{I D}, \operatorname{Cert}_{I D}, \perp, 0\right)$ in $\mathcal{L}_{2}$. For the two cases, $\mathcal{C}$ will update $H \leftarrow H+1$. We suppose that the other oracles defined later only respond to the identity which has been created.

(2) $\mathcal{O}$-Publickey: For a handle $H$, the challenger looks up the identity $I D$ in the list $\mathcal{L}_{1}$ or $\mathcal{L}_{2}$ and returns the public key $p k_{I D}$ to the adversary $\mathcal{A}_{1}$ or $\mathcal{A}_{2}$.

(3) $O$-Replacepublickey: The adversary $\mathcal{A}_{1}$ can replace the public key $p k_{I D}$ of the identity $I D$ with a new public key $p k_{I D}^{\prime}$ of its choice. In order to ensure that the public key $p k_{I D}^{\prime}$ is valid, the challenger runs SetPrivateKey $(I D, m p k) \rightarrow s k_{I D}$ and adds an item $\left(H, I D, I D^{\prime}, p k_{I D}^{\prime}, \perp, \perp, \perp\right.$, 
$0,0)$ in the list $\mathcal{L}_{1}$. It updates $H \leftarrow H+1$. The constraint is that for the challenge identity $I D^{*}$ the adversary $\mathcal{A}_{1}$ isn't allowed to replace the public key before the challenge phase and at the same time queries the certificate at some point. Thus $\mathcal{A}_{1}$ obtains a challenge ciphertext about a public key on which he calculates the decryption key.

(4) $\mathcal{O}$-Certificate: For a handle $H$, the challenger looks up the identity $I D$ in the list $\mathcal{L}_{1}$. The challenger calculates: $\bar{H}\left(I D, p k_{I D}\right) \rightarrow I D^{\prime}, \operatorname{SetCertificate}\left(I D^{\prime}, p k_{I D}, m p k, m s k\right) \rightarrow \operatorname{Cert}_{I D}$. It returns the certificate $\operatorname{Cert}_{I D}$ to the adversary $\mathcal{A} 1$ and updates the item $\left(H, I D, I D^{\prime}, p k_{I D}, s k_{I D}, \perp\right.$, $\perp, 0,0)$ with $\left(H, I D, I D^{\prime}, p k_{I D}, s k_{I D}, \operatorname{Cert}_{I D}, \perp, 0,0\right)$.

(5) $\mathcal{O}$-Decryptionkey: The query can be done for the identity that his public key is not replaced. If an adversary queries the decryption key for the identity $I D$, the challenger $\mathcal{C}$ scans the list $\mathcal{L}_{1}$ or $\mathcal{L}_{2}$ to find $s k_{I D}$ and $\operatorname{Cert}_{I D}$. Then it calculates the decryption key: $\operatorname{SetDecryptKey}\left(s k_{I D}\right.$, $\left.\operatorname{Cert}_{I D}\right) \rightarrow d k_{I D}$. For $\mathcal{A} 1, \mathcal{C}$ outputs $d k_{I D}$ to $\mathcal{A}_{1}$ and updates the item $\left(H, I D, I D^{\prime}, p k_{I D}\right.$, $\left.s k_{I D}, \operatorname{Cert}_{I D}, \perp, 0,0\right)$ with $\left(H, I D, I D^{\prime}, p k_{I D}, s k_{I D}, \operatorname{Cert}_{I D}, d k_{I D}, 0,0\right)$. For $\mathcal{A} 2, \mathcal{C}$ outputs $d k_{I D}$ to it and updates the item $\left(H, I D, I D^{\prime}, p k_{I D}, s k_{I D}, \operatorname{Cert}_{I D}, \perp, 0\right) \quad$ with $\left(H, I D, I D^{\prime}, p k_{I D}, s k_{I D}, \operatorname{Cert}_{I D}, d k_{I D}, 0\right)$.

(6) $\mathcal{O}$-Decrypt : If the adversary queries a decryption for $(I D, C)$, the challenger scans the list $\mathcal{L}_{1}$ or $\mathcal{L}_{2}$ to get the decryption key $d k_{I D}$. The challenger invokes the algorithm Decrypt to obtain the corresponding plaintext $M$ and gives it to the adversary $\mathcal{A}_{1}$ or $\mathcal{A}_{2}$.

(7) $\mathcal{O}$-Leakdecryptionkey: Given a handle $H$ and a leakage function $f$ with the output of constant size, the challenger $\mathcal{C}$ looks up the item $\left(H, I D, I D^{\prime}, p k_{I D}, s k_{I D}, C_{e r t}, d k_{I D}, l_{d k}, l_{m s k}\right)$ or $\left(H, I D, I D^{\prime}, p k_{I D}, s k_{I D}, \operatorname{Cert}_{I D}, d k_{I D}, l_{d k}\right)$ which includes $H$ in list $\mathcal{L}_{1}$ or $\mathcal{L}_{2} . l_{d k}$ and $l_{m s k}$ are initially zero. $\mathcal{C}$ judges if $l_{d k}+\left|f\left(d k_{I D}\right)\right| \leq \lambda_{d k}$ where $\lambda_{d k}$ is the leakage bound of the decryption key. If this is true, the challenger returns the output of $f\left(d k_{I D}\right)$ to $\mathcal{A}_{1}$ or $\mathcal{A}_{2}$ and updates $l_{d k}$ with $l_{d k}+\left|f\left(d k_{I D}\right)\right|$ in list $\mathcal{L}_{1}$ or $\mathcal{L}_{2}$.

(8) O-Leakmasterkey: Given a handle $H$ and a leakage function $f$ with the output of constant size, the challenger looks up the item $\left(H, I D, I D^{\prime}, p k_{I D}, s k_{I D}, C_{e r t}{ }_{I D}, d k_{I D}, l_{d k}, l_{m s k}\right)$ which includes $H$ in list $\mathcal{L}_{1}$. It judges if $l_{m s k}+|f(m s k)| \leq \lambda_{m s k}$ where $\lambda_{m s k}$ is the leakage bound of the master secret key. If this is true, the challenger returns the output of $f(m s k)$ to $\mathcal{A}_{1}$ and updates $l_{m s k}$ with $l_{m s k}+|f(m s k)|$ in list $\mathcal{L}_{1}$.

\subsection{Security Model of LR-CBE}

In our model there are two type adversaries, so the security is obtained from two security games. The challenger plays the games with the adversary $\mathcal{A}_{1}$ or $\mathcal{A}_{2}$.

\subsubsection{Security against Type I Adversary}

The security of LR-CBE against $\mathcal{A}_{1}$ is defined by the game $\mathcal{T}_{1}$ Game $R$ which is played between the challenger and the adversary $\mathcal{A}_{1}$. $\mathcal{T}_{1}$ _Game $R_{-}$is defined as follows.

$\mathcal{T}_{1}$ Game_R :

Initialize: The challenger invokes the algorithm Setup to generate the master secret key and the master public key: $\operatorname{Setup}\left(1^{\vartheta}\right) \rightarrow(m p k, m s k)$. The challenger keeps the master secret key as secret and issues the master public key to all users.

Phase 1: The adversary queries the oracles: $\mathcal{O}$-Create, $\mathcal{O}$-PublicKey, $\mathcal{O}$-Leakdecryptionkey, 
$\mathcal{O}$-LeakMasterKey, $\mathcal{O}$-Certificate, $\mathcal{O}$-Replacepublickey, $\mathcal{O}$-Decryptionkey, $\mathcal{O}$-Decrypt

The constraints are as follows.

$\mathcal{A}_{1}$ can not query the decryption key for the challenge identity $I D^{*}$. For the challenger identity $I D^{*}$, if $\mathcal{A}_{1}$ replaces the public key, he is not allowed to query the corresponding certificate.

Challenge: The adversary $\mathcal{A}_{1}$ gives two equal length messages $M_{0}, M_{1} \in \mathcal{M}$ and an identity $I D^{*}$ to the challenger. $\mathcal{M}$ is a given message space. The challenger looks up the corresponding item consisting of the $I D^{*}$ in list $\mathcal{L}_{1}$. If the item is not in list $\mathcal{L}_{1}$, the challenger will make create query $\mathcal{O}$-Create for the identity $I D^{*}$ firstly. Then the challenger randomly selects a bit $\xi \in\{0,1\}$ and runs algorithm Encrypt to generate the ciphertext $C^{*}$ about the message $M_{\xi}$ : $\operatorname{Encrypt}\left(I D^{*}, m p k, M_{\xi}, p k_{I D^{*}}\right) \rightarrow C^{*}$. At last, the challenger sends the ciphertext $C^{*}$ to $\mathcal{A}_{1}$.

Phase 2: Just similar to Phase 1, $\mathcal{A} 1$ queries the oracles: $\mathcal{O}$-Create, $\mathcal{O}$-Publickey, $\mathcal{O}$-Decrypt, $\mathcal{O}$-Replacepublickey, $\mathcal{O}$-Certificate, $\mathcal{O}$-Decryptionkey. The basic constraints are the same as that of Phase 1 . The other constraints are that these oracles can not query the challenge identity $I D^{*}$. Furthermore, no leakage query is allowed in this phase. If we allow leakage queries, the adversary can encode the Decrypt algorithm of $C^{*}$ as a leakage function and wins the game trivially.

Guess: At last, $\mathcal{A}_{1}$ guesses a bit $\xi^{\prime} \in\{0,1\}$. If $\xi^{\prime}=\xi, \mathcal{A}_{1}$ wins the game.

The advantage that an adversary $\mathcal{A}_{1}$ wins this game is $A d v_{\mathcal{A}_{1}}^{\mathcal{T}_{1} \text { Game }{ }_{-}}\left(\lambda_{d k}, \lambda_{m s k}\right)=\left|\operatorname{Pr}\left[\xi^{\prime}=\xi\right]-\frac{1}{2}\right|$.

Definition 4: If there is no PPT adversary $\mathcal{A} 1$ who can win $\mathcal{T}_{1}$ Game $R_{-}$with non-negligible advantage $\left(A d v_{\mathcal{A}_{1}}^{T_{1} \text { Game }}{ }^{R}\left(\lambda_{d k}, \lambda_{m s k}\right) \leq \varepsilon\right.$ where $\varepsilon$ is a negligible value), the LR-CBE is called type I secure against the adaptive chosen ciphertext attacks.

\subsubsection{Security against Type II Adversary}

The security of LR-CBE against $\mathcal{A}_{2}$ is defined by the game $\mathcal{T}_{2}$ _Game $R_{-}$which is played between the challenger and the adversary $\mathcal{A}_{2}$. $\mathcal{T}_{2}$ _Game $R$ is defined as follows.

$\mathcal{T}_{2}$ Game_R:

Initialize: The challenger invokes the algorithm Setup to generate the master public key and the master secret key: $\operatorname{Setup}\left(1^{\vartheta}\right) \rightarrow(m p k, m s k)$. The challenger issues the master public key and the master secret key to $\mathcal{A} 2$.

Phase 1: The adversary may query the oracles: $\mathcal{O}$-Create, $\mathcal{O}$-Publickey, $\mathcal{O}$-Decryptionkey, $\mathcal{O}$-Decrypt, $\mathcal{O}$-Leakdecryptionkey. Because $\mathcal{A}_{2}$ knows the master secret key it does not need to query the oracle $\mathcal{O}$-Leakmasterkey and $\mathcal{O}$-Certificate. The constraints are as follows.

$\mathcal{A}_{2}$ can not query the decryption key for the challenge identity $I D^{*} . \mathcal{A}_{2}$ is not allowed to replace the public key at any point.

Challenge: The adversary $\mathcal{A}_{2}$ gives two equal length messages $M_{0}, M_{1} \in \mathcal{M}$ and an identity $I D^{*}$ to the challenger. $\mathcal{M}$ is a given message space. The challenger looks up the corresponding item consisting of the $I D^{*}$ in list $\mathcal{L}_{2}$. If the item is not in list $\mathcal{L}_{2}$, The challenger will make create query $\mathcal{O}$-Create for the identity $I D^{*}$.

Then the challenger randomly selects a bit $\xi \in\{0,1\}$ and runs algorithm Encrypt to get the ciphertext $C^{*}$ about the message $M_{\xi}: \operatorname{Encrypt}\left(I D^{*}, m p k, M_{\xi}, p k_{I D^{*}}\right) \rightarrow C^{*}$. At last, the challenger sends the ciphertext $C^{*}$ to the adversary $\mathcal{A}_{2}$.

Phase 2: Just similar to Phase 1, $\mathcal{A}_{2}$ queries the oracles: $\mathcal{O}$-Create, $\mathcal{O}$-Publickey, $\mathcal{O}$-Decrypt, $\mathcal{O}$-Decryptionkey. The basic constraints are the same as that of Phase 1 . The other constraints are 
that these oracles can not query the challenge identity $I D^{*}$. Furthermore, no leakage query is allowed in this phase. If we allow leakage queries, the adversary can encode the Decrypt algorithm of $C^{*}$ as a leakage function and wins the game trivially.

Guess: At last, $\mathcal{A}_{2}$ guesses a bit $\xi^{\prime} \in\{0,1\}$. If $\xi^{\prime}=\xi, \mathcal{A}_{2}$ wins the game.

The advantage that an adversary $\mathcal{A}_{2}$ wins this game is $A d v_{\mathcal{A}_{2}}^{\mathcal{T}_{2} \text { Game }{ }_{-} R}\left(\lambda_{d k}\right)=\left|\operatorname{Pr}\left[\xi^{\prime}=\xi\right]-\frac{1}{2}\right|$.

Definition 5: If there is no PPT adversary $\mathcal{A}_{2}$ who wins $\mathcal{T}_{2}$ _Game $R$ with non-negligible advantage $\left(A d v_{\mathcal{A}_{2}}^{\mathcal{T}_{2} \text { Game }{ }_{-} R}\left(\lambda_{d k}\right) \leq \varepsilon\right)$, the LR-CBE is called type II secure against the adaptive chosen ciphertext attacks.

\section{Construction of our LR-CBE}

We firstly give an NIZK proof system $\Pi=($ Gen, $\operatorname{Prf}$, Ver $)$ which will be employed in our scheme. We define $\Pi=($ Gen $, \operatorname{Prf}, \operatorname{Ver})$ is an NIZK proof system with the language $\mathrm{L}=\left\{\beta: Y^{\beta}=Z\right\}$ where $\beta \in Z_{N}$, and $Y, Z \in G_{T} . \bar{H}: \mathcal{I D} \times \mathcal{P K} \rightarrow \mathcal{I D}$ is a hash function, where $\mathcal{I D}$ is the identity space and $\mathcal{P K}$ is the public key space. The hash function is used to maintain the security when a CLE is converted to a CBE (Please refer to [28]). Suppose that any identity is an element of $Z_{N}$. Our LR-CBE consists of the following seven algorithms.

Setup: It firstly creates composite order bilinear groups $\left(N=p_{1} p_{2} p_{3}, G, G_{T}, e\right)$. Then, it randomly selects $g_{1}, u_{1}, h_{1}, v_{1} \in G_{p_{1}}$ and $g_{3} \in G_{p_{3}}$. It runs algorithm $G e n$ of $\Pi$ to generate the common reference string $\operatorname{crs}$ and selects random $\left(\alpha, x_{1}, x_{2}, \ldots, x_{n}, r, y_{1}, y_{2}, \ldots, y_{n}\right) \in Z_{N}^{2 n+2}$ and a vector $\vec{\rho}=<\rho_{1}, \rho_{2}, \ldots, \rho_{n+3}>\in Z_{N}^{n+3}$ where $n \geq 2$ is an integer. The value of $n$ can be varied. A bigger value of $n$ will generate a bigger leakage rate. Leakage rate is the value that the number of leaked bits from the decryption key or the master secret key divides by the number of bits for the decryption key or master secret key. A smaller value of $n$ will lead to a smaller master public key. It outputs the master public key $m p k=\left(N, G, G_{T}, e, e\left(g_{1}, v_{1}\right)^{\alpha}, g_{1}, g_{1}^{x_{1}}, \ldots, g_{1}^{x_{n}}, u_{1}, h_{1}, v_{1}, g_{3}, c r s\right)$ and the master secret key $m s k=\left(\vec{K}, K_{1}, K_{2}, K_{3}\right)=\left(<v_{1}^{y_{1}}, \ldots, v_{1}^{y_{n}}>, g_{1}^{\alpha} h_{1}^{-r} \prod_{i=1}^{n} g_{1}^{-x_{i} y_{i}}, v_{1}^{r}, u_{1}^{r}\right) * g_{3}^{\vec{\rho}}$.

SetPrivateKey: The user sets the private key $s k_{I D}=\beta$ where $\beta \in Z_{N}$.

SetPublicKey: The user sets public key $p k_{I D}=(Y, \pi)=\left(e\left(g_{1}, v_{1}\right)^{\alpha \beta}, \pi\right)$ where $\pi \leftarrow \operatorname{Prf}(\operatorname{crs}$, $\left.\left(e\left(g_{1}, v_{1}\right)^{\alpha \beta}, e\left(g_{1}, v_{1}\right)^{\alpha}\right), \beta\right)$ is an NIZK proof that $\beta$ is the discrete logarithm of $e\left(g_{1}, v_{1}\right)^{\alpha \beta}$ to the base $e\left(g_{1}, v_{1}\right)^{\alpha}$.

SetCertificate: The CA randomly selects a vector $\vec{\rho}^{\prime}=<\rho_{1}^{\prime}, \rho_{2}^{\prime}, \ldots, \rho_{n+2}^{\prime}>\in Z_{N}^{n+2}$ and $n+1$ elements $\left(r^{\prime}, z_{1}, \ldots, z_{n}\right) \in Z_{N}^{n+1}$. Then, it calculates the certificate as follows: $\bar{H}\left(I D, p k_{I D}\right)=I D^{\prime}$, $\operatorname{Cert}_{I D}=\left(\vec{D}, D_{1}, D_{2}\right)=\left(\vec{K}, K_{1}, K_{2}\right) *\left(<v_{1}^{z_{1}}, \ldots, v_{1}^{z_{n}}>,\left(K_{3}\right)^{-I D^{\prime}}\left(u_{1}^{I D^{\prime}} h_{1}\right)^{r^{\prime}} \prod_{i=1}^{n} g_{1}^{-x_{i} z_{i}}, v_{1}^{r^{\prime}}\right) * g_{3}^{\vec{\rho}^{\prime}}$. The $G_{p_{1}}$ part of $\operatorname{Cert}_{I D}$ can be viewed as $\left.\left(<v_{1}^{z_{1}^{\prime}}, \ldots, v_{1}^{z_{n}^{\prime}}\right\rangle, g_{1}^{\alpha}\left(u_{1}^{I D^{\prime}} h_{1}\right)^{-r^{\prime \prime}} \prod_{i=1}^{n} g_{1}^{-x_{i} z_{i}^{\prime}}, v_{1}^{r^{\prime \prime}}\right)$ for some $\left(r^{\prime \prime}, z_{1}^{\prime}, \ldots, z_{n}^{\prime}\right) \in Z_{N}^{n+1}$ where $z_{i}^{\prime}=y_{i}+z_{i}$ for $i=1$ to $n$ and $r^{\prime \prime}=r+r^{\prime}$.

Encrypt: The sender verifies the validation for proof $\pi$. If $\pi$ is valid, it picks randomly $s \in Z_{n}$ and computes the ciphertext: $C=\left(C_{0}, \vec{C}, C_{1}, C_{2}\right)=\left(M . e\left(g_{1}, v_{1}\right)^{\alpha \beta s},<g_{1}^{x_{1} s}, \ldots, g_{1}^{x_{n} s}>, v_{1}^{s},\left(u_{1}^{I D^{\prime}} h_{1}\right)^{s}\right)$, 
where $I D^{\prime}=\bar{H}\left(I D, p k_{I D}\right)$.

SetDecryptKey: The user picks $\vec{\rho}^{\prime \prime}=<\rho_{1}^{\prime \prime}, \ldots, \rho_{n+2}^{\prime \prime}>\in Z_{N}^{n+2}, \vec{w}=<w_{1}, \ldots, w_{n}>\in Z_{N}^{n}$ and $t \in Z_{N}$ randomly. The user calculates the decryption key as follows: $\bar{H}\left(I D, p k_{I D}\right)=I D^{\prime}, d k_{I D}=(\vec{S}$, $\left.S_{1}, S_{2}\right)=\left(\vec{D}, D_{1}, D_{2}\right)^{\beta} *\left(<v_{1}^{w_{1}}, \ldots, v_{1}^{w_{n}}>,\left(u_{1}^{I D^{\prime}} h_{1}\right)^{-t} \prod_{i=1}^{n} g_{1}^{-x_{i} w_{i}}, v_{1}^{t}\right) * g_{3}^{\vec{\rho}^{\prime \prime}}$. The $G_{p_{1}}$ part of $d k_{I D}$ can be viewed as $\left(<v_{1}^{w_{1}^{\prime}}, \ldots, v_{1}^{w_{n}^{\prime}}>, g_{1}^{\alpha \beta}\left(u_{1}^{I D^{\prime}} h_{1}\right)^{-t^{\prime}} \prod_{i=1}^{n} g_{1}^{-x_{i} w_{i}^{\prime}}, v_{1}^{t^{\prime}}\right)$ for some $\left(t^{\prime}, w_{1}^{\prime}, \ldots, w_{n}^{\prime}\right) \in Z_{N}^{n+1}$ where $w_{i}^{\prime}=w_{i}+\left(y_{i}+z_{i}\right)^{\beta}$ for $i=1$ to $n$ and $t^{\prime}=t+\left(r+r^{\prime}\right)^{\beta}$.

Decrypt: By using the decryption key, the user gets $M=\frac{C_{0}}{e(\vec{C}, \vec{S}) \cdot e\left(C_{1}, S_{1}\right) \cdot e\left(C_{2}, S_{2}\right)}$.

Correctness.

$$
\begin{aligned}
& e(\vec{C}, \vec{S}) \cdot e\left(C_{1}, S_{1}\right) \cdot e\left(C_{2}, S_{2}\right) \\
& =e\left(<g_{1}^{x_{1} s}, \ldots, g_{1}^{x_{n} s}>,<v_{1}^{w_{1}^{\prime}}, \ldots, v_{1}^{w_{n}^{\prime}}>^{*}<g_{3}^{\left(\rho_{1}+\rho_{1}^{\prime}\right)^{\beta}+\rho_{1}^{\prime \prime}}, \ldots, g_{3}^{\left(\rho_{n}+\rho_{n}^{\prime}\right)^{\beta}+\rho_{n}^{\prime \prime}}>\right) \text {. } \\
& e\left(v_{1}^{s}, g_{1}^{\alpha \beta}\left(u_{1}^{I D^{\prime}} h_{1}\right)^{-t^{\prime}} \prod_{i=1}^{n} g_{1}^{-x_{i} w_{i}^{\prime}} \cdot g_{3}^{\rho_{n+1}^{\prime \prime}}\right) \cdot e\left(\left(u_{1}^{I D^{\prime}} h_{1}\right)^{s}, v_{1}^{t^{\prime}} \cdot g_{3}^{\rho_{n+2}^{\prime \prime}}\right) \\
& =e\left(<g_{1}^{x_{1} s}, \ldots, g_{1}^{x_{n} s}>,<v_{1}^{w_{1}^{\prime}} \cdot g_{3}^{\left(\rho_{1}+\rho_{1}^{\prime}\right)^{\beta}+\rho_{1}^{\prime \prime}}, \ldots, v_{1}^{w_{n}^{\prime}} \cdot g_{3}^{\left(\rho_{n}+\rho_{n}^{\prime}\right)^{\beta}+\rho_{n}^{\prime \prime}}>\right) \text {. } \\
& e\left(v_{1}^{s}, g_{1}^{\alpha \beta}\left(u_{1}^{I D^{\prime}} h_{1}\right)^{-t^{\prime}} \prod_{i=1}^{n} g_{1}^{-x_{i} w_{i}^{\prime}} \cdot g_{3}^{\rho_{n+1}^{\prime \prime}}\right) \cdot e\left(\left(u_{1}^{I D^{\prime}} h_{1}\right)^{s}, v_{1}^{t^{\prime}} \cdot g_{3}^{\rho_{n+2}^{\prime \prime}}\right) \\
& =\prod_{i=1}^{n} e\left(g_{1}^{x_{i} s}, v_{1}^{w_{i}^{\prime}} \cdot g_{3}^{\left(\rho_{i}+\rho_{i}^{\prime}\right)^{\beta}+\rho_{i}^{\prime \prime}}\right) \cdot e\left(v_{1}^{s}, g_{1}^{\alpha \beta}\left(u_{1}^{I D^{\prime}} h_{1}\right)^{-t^{\prime}} \prod_{i=1}^{n} g_{1}^{-x_{i} w_{i}^{\prime}} \cdot g_{3}^{\rho_{n+1}^{\prime \prime}}\right) \cdot e\left(\left(u_{1}^{I D^{\prime}} h_{1}\right)^{s}, v_{1}^{t^{\prime}} \cdot g_{3}^{\rho_{n+2}^{\prime \prime}}\right) \\
& =\prod_{i=1}^{n} e\left(g_{1}^{x_{i} s}, v_{1}^{w_{i}^{\prime}}\right) \cdot \prod_{i=1}^{n} e\left(g_{1}^{x_{i} s}, g_{3}^{\left(\rho_{i}+\rho_{i}^{\prime}\right)^{\beta}+\rho_{i}^{\prime \prime}}\right) \cdot e\left(v_{1}^{s}, g_{1}^{\alpha \beta}\left(u_{1}^{I D^{\prime}} h_{1}\right)^{-t^{\prime}} \prod_{i=1}^{n} g_{1}^{-x_{i} w_{i}^{\prime}}\right) \cdot e\left(v_{1}^{s}, g_{3}^{\rho_{n+1}^{\prime \prime}}\right) \text {. } \\
& e\left(\left(u_{1}^{I D^{\prime}} h_{1}\right)^{s}, v_{1}^{t^{\prime}}\right) \cdot e\left(\left(u_{1}^{I D^{\prime}} h_{1}\right)^{s}, g_{3}^{\rho_{n+2}^{\prime \prime}}\right) \\
& =\prod_{i=1}^{n} e\left(g_{1}^{x_{i} s}, v_{1}^{w_{i}^{\prime}}\right) \cdot e\left(v_{1}^{s}, g_{1}^{\alpha \beta}\left(u_{1}^{I D^{\prime}} h_{1}\right)^{-t^{\prime}} \prod_{i=1}^{n} g_{1}^{-x_{i} w_{i}^{\prime}}\right) \cdot e\left(\left(u_{1}^{I D^{\prime}} h_{1}\right)^{s}, v_{1}^{t^{\prime}}\right) \\
& =\prod_{i=1}^{n} e\left(g_{1}^{x_{i} s}, v_{1}^{w_{i}^{\prime}}\right) \cdot e\left(v_{1}^{s}, \prod_{i=1}^{n} g_{1}^{-x_{i} w_{i}^{\prime}}\right) \cdot e\left(v_{1}^{s}, g_{1}^{\alpha \beta}\right) \cdot e\left(v_{1}^{s},\left(u_{1}^{I D^{\prime}} h_{1}\right)^{-t^{\prime}}\right) \cdot e\left(\left(u_{1}^{I D^{\prime}} h_{1}\right)^{s}, v_{1}^{t^{\prime}}\right) \\
& =\prod_{i=1}^{n} e\left(g_{1}^{x_{i} w_{i}^{\prime}}, v_{1}^{s}\right) \cdot e\left(v_{1}^{s}, \prod_{i=1}^{n} g_{1}^{-x_{i} w_{i}^{\prime}}\right) \cdot e\left(v_{1}, g_{1}\right)^{\alpha \beta s} \cdot e\left(v_{1}^{t^{\prime}},\left(u_{1}^{I D^{\prime}} h_{1}\right)^{-s}\right) \cdot e\left(\left(u_{1}^{I D^{\prime}} h_{1}\right)^{s}, v_{1}^{t^{\prime}}\right) \\
& =e\left(\prod_{i=1}^{n} g_{1}^{x_{i} w_{i}^{\prime}}, v_{1}^{s}\right) \cdot e\left(v_{1}^{s}, \prod_{i=1}^{n} g_{1}^{-x_{i} w_{i}^{\prime}}\right) \cdot e\left(v_{1}, g_{1}\right)^{\alpha \beta s} \\
& =e\left(v_{1}, g_{1}\right)^{\alpha \beta s}
\end{aligned}
$$

\section{Security Proof}

Inspired by dual system encryption method [26, 29, 30], we uses semi-functional ciphertexts and keys in our proof. In order to accomplish our proof, we give dual system construction of our LR-CBE.

\subsection{Dual System Description of Our LR-CBE}

DS-Setup: The algorithm is based on Setup. It outputs a normal master public key and a semi-functional master secret key $\widetilde{m s k}$.

DS-SetCertificate: The algorithm is based on SetCertificate. It is run by the certificate authority. It 
takes as input master public key $m p k$, the SF master secret key $\widetilde{m s k}$, the identity $I D$ and the corresponding public $p k_{I D}$. It outputs the user's SF certificate $\overline{\operatorname{Cert}_{I D}}$.

DS-SetDecryptKey: The algorithm is run by the user $I D$. It takes as input the master public key. It outputs the user's semi-functional decryption key $\widetilde{d k_{I D}}$.

DS-Encrypt: The algorithm is run by the sender. It takes as input the master public key $m p k$, the plaintext $M$, the receiver's identity $I D$ and the corresponding public $p k_{I D}$. It outputs the SF ciphertext $\widetilde{C}$.

The SF decryption keys can only decrypt normal ciphertexts. The normal decryption keys can decrypt normal and semi-functional ciphertexts. Of course, if the input of the algorithm SetCertificate is the SF master secret key $\widetilde{m s k}$, the output of SetCertificate is SF certificate $\widetilde{\text { Cert }_{I D}}$. If the input of the algorithm SetDecryptKey is SF certificate $\overline{\operatorname{Cert}_{I D}}$, the output of the algorithm SetDecryptKey is SF decryption key $\widetilde{d k_{I D}}$.

Here, we use $\widetilde{X}$ to denote the semi-functional construction of $X$. When the semantic context is clear, we also use $X$ to denote the corresponding semi-functional construction.

\subsection{Dual System Construction of Our LR-CBE}

In section 4, keys and ciphertexts are normal. That is to say, they don't contain $G_{p_{2}}$ part. Here, we give the dual system construction of our LR-CBE according to the description in Subsection 5.1.

DS-Setup: The algorithm invokes Setup to generate a normal master secret key $m s k=\left(\vec{K}, K_{1}\right.$, $\left.K_{2}, K_{3}\right)$. It selects $\vec{\imath} \in Z_{N}^{n},\left(l_{1}, l_{2}, l_{3}\right) \in Z_{N}^{3}$ randomly, then computes the semi-functional master secret key $\widetilde{m s k}=\left(\vec{K} * g_{2}^{\vec{i}}, K_{1} \cdot g_{2}^{l_{1}}, K_{2} \cdot g_{2}^{l_{2}}, K_{3} \cdot g_{2}^{l_{3}}\right)$.

DS-SetCertificate: The algorithm runs SetCertificate to generate the normal certificate $\operatorname{Cert}_{I D}=\left(\vec{D}, D_{1}, D_{2}\right)$. It selects $\vec{\eta} \in Z_{N}^{n},\left(\eta_{1}, \eta_{2}\right) \in Z_{N}^{2}$ randomly, then computes the semi-functional certificate $\overline{\operatorname{Cert}_{I D}}=\left(\vec{D} * g_{2}^{\vec{\eta}}, D_{1} \cdot g_{2}^{\eta_{1}}, D_{2} \cdot g_{2}^{\eta_{2}}\right)$.

DS-SetDecryptKey: The algorithm runs SetDecryptKey to generate the normal decryption key $d k_{I D}=\left(\vec{S}, S_{1}, S_{2}\right)$. Next, it randomly selects $\vec{\theta} \in Z_{N}^{n},\left(\theta_{1}, \theta_{2}\right) \in Z_{N}^{2}$ and computes the semi functional decryption key $\widetilde{d k_{I D}}=\left(\vec{S} * g_{2}^{\vec{\theta}}, S_{1} \cdot g_{2}^{\theta_{1}}, S_{2} \cdot g_{2}^{\theta_{2}}\right)$.

DS-Encrypt: The algorithm invokes Encrypt to get normal ciphertext $C=\left(C_{0}, \vec{C}, C_{1}, C_{2}\right)$. Next, it selects $\vec{\delta} \in Z_{N}^{n},\left(\delta_{1}, \delta_{2}\right) \in Z_{N}^{2}$ randomly and computes the semi-functional ciphertext $\widetilde{C}=\left(C_{0}\right.$, $\left.\vec{C} * g_{2}^{\vec{\delta}}, C_{1} \cdot g_{2}^{\delta_{1}}, C_{2} \cdot g_{2}^{\delta_{2}}\right)$.

If $\vec{\theta} \cdot \vec{\delta}+\theta_{1} \delta_{1}+\theta_{2} \delta_{2}=0 \bmod p_{2}$, the SF decryption key is called as nominal semi-functional (NSF) decryption key. In this case, even if the ciphertext is semi functional, Decrypt will be done successfully. Otherwise, we call the SF decryption key as true SF decryption key.

\subsection{Security of Our LR-CBE}

\subsubsection{Security Proof against Type I Adversary}

In order to prove the security with leakage resilience, we give a series of games here, which are modifications of the game $\mathcal{T}_{1}$ Game $R$. If we prove that these games are indistinguishable we finish the security proof of our scheme. We use $Q$ to denote the maximum number of $\mathcal{O}$-Create queries in the games.

These additional games are as follows. 
$\mathcal{T}_{1}$ Game_0 $:$ It is nearly the same as $\mathcal{T}_{1}$ Game $R$ besides the challenge ciphertext. In $\mathcal{T}_{1}$ Game_0 the challenge ciphertext is semi-functional.

$\mathcal{T}_{1}$ Game_k $(k=1$ to $Q)$ : The challenge ciphertext is semi-functional. For the adversary's queries, the challenger answers in two ways. Toward the first $k$ queries, the challenger does as follows.

If the adversary makes a certificate query or decryption key query, the challenger creates the SF certificate and SF decryption key. If the adversary makes a replace public key query, the challenger only creates the SF certificate. The challenger adds the corresponding item in list $\mathcal{L}_{1}$ and gives it to the adversary.

For the remaining queries, the challenger creates normal certificate and normal decryption key. $\mathcal{T}_{1}$ Game_Msk : It is nearly the same as $\mathcal{T}_{1}$ Game $Q$ except that in $\mathcal{T}_{1}$ Game Gsk the master secret key is semi-functional. Thus, for $\mathcal{O}$-Leakmasterkey query, the challenger creates semi-functional master secret key and sends the output of the leakage function to the adversary. The leakage function takes the semi-functional master secret key as input.

$\mathcal{T}_{1}$ _Game_Final : It is nearly the same as $\mathcal{T}_{1}$ Game_Msk except that the challenger randomly selects a message $M_{\varsigma}$ and encrypts it. In $\mathcal{T}_{1}$ Game_Msk, the challenger encrypts a challenge message $M_{\xi}$. From the adversary's point of view, in $\mathcal{T}_{1}$ Game_Final the bit $\xi^{\prime}$ of his choice is independent of the challenger's bit $\xi$.

In the following table 1, we explain the types of master secret key, ciphertexts and decryption keys which are created in different games. Let SF denote the semi-functional key or ciphertext. Let $\mathrm{N}$ denote the normal key or ciphertext. We use $T_{M}, T_{C}$ and $T_{D}$ to denote the types of master secret key, ciphertext and decryption key, respectively. In each game, the maximum number of create query $\mathcal{O}$-Create is $Q$. Thus, we use $(\underbrace{\left(\mathrm{T}_{\mathrm{M}}, \mathrm{T}_{\mathrm{C}}, \mathrm{T}_{\mathrm{D}}\right), \ldots,\left(\mathrm{T}_{\mathrm{M}}, \mathrm{T}_{\mathrm{C}}, \mathrm{T}_{\mathrm{D}}\right)}_{Q})$ to denote the according types of $Q$ create queries in a game. For every game above, the types of the ciphertexts are the same in every create query. At the same time, the types of master keys are the same in every create query. Thus, $\left(\left(\mathrm{T}_{\mathrm{M}}, \mathrm{T}_{\mathrm{C}}, \mathrm{T}_{\mathrm{D}}\right), \ldots,\left(\mathrm{T}_{\mathrm{M}}, \mathrm{T}_{\mathrm{C}}, \mathrm{T}_{\mathrm{D}}\right)\right)$ could be shortened as $\left(\mathrm{T}_{\mathrm{M}}, \mathrm{T}_{\mathrm{C}},(\underbrace{\mathrm{T}_{\mathrm{D}}, \ldots \mathrm{T}_{\mathrm{D}}}_{Q})\right)$ to denote the according types of $Q$ create queries in a game.

Table 1. The types of master secret keys, ciphertexts and decryption keys in different games.

\begin{tabular}{|c|c|}
\hline Games & $\begin{array}{r}\text { The type of master secret keys, ciphertexts and decryption keys: } \\
\left(\mathrm{T}_{\mathrm{M}}, \mathrm{T}_{\mathrm{C}},\left(\mathrm{T}_{\mathrm{D}}, \ldots, \mathrm{T}_{\mathrm{D}}\right)\right)\end{array}$ \\
\hline $\mathcal{T}_{1} \_$Game_R & $(\mathrm{N}, \mathrm{N},(\mathrm{N}, \ldots, \mathrm{N}))$ \\
\hline $\mathcal{T}_{1} \_$Game_o & $(\mathrm{N}, \mathrm{SF},(\mathrm{N}, \ldots \ldots, \mathrm{N}))$ \\
\hline $\mathcal{T}_{1}$ Game_k & $\left(\mathrm{N}, \mathrm{SF},\left(\mathrm{SF}, \ldots, \mathrm{SF}_{k}, \mathrm{~N}, \ldots, \mathrm{N}\right)\right)$ \\
$k \in(1, \ldots, Q-1)$ & $(\mathrm{N}, \mathrm{SF},(\mathrm{SF}, . ., \mathrm{SF}))$ \\
\hline $\mathcal{T}_{1}$ Game_Q & $(\mathrm{SF}, \mathrm{SF},(\mathrm{SF}, \ldots, \mathrm{SF}))$ \\
\hline $\mathcal{T}_{1}$ Game_Msk & $(\mathrm{SF}, \mathrm{SF},(\mathrm{SF}, \ldots, \mathrm{SF}))$ \\
\hline $\mathcal{T}_{1}$ Game_Final &
\end{tabular}

Theorem 1. Under the assumption 1, assumption 2 and assumption 3, for $\lambda_{d k}=(n-2 c-1) \lambda$ bits leakage of the decryption key and $\lambda_{m s k}=(n-2 c-1) \lambda$ bits leakage of the master secret key, the LR-CBE scheme is secure against $\mathcal{A}_{1}$ where $n \geq 2$ is an integer and $c$ is a fixed positive constant.

The value of $n$ can be varied. A larger value of $n$ will generate a larger leakage rate. The smaller value of $n$ will lead to a smaller master public key. The concrete explanation is given in Section 6 .

Proof. The general idea of proof is as follows. We will use a series of games $\mathcal{T}_{1}$ Game $R$, $\mathcal{T}_{1}$ _Game_k $(k \in(0,1, \ldots, Q)), \mathcal{T}_{1}$ _Game_Msk and $\mathcal{T}_{1}$ _Game_Final and Lemma 1 to 
Lemma 9 to finish our security proof. On one hand, we prove that these games are indistinguishable by Lemma 2 to Lemma 9. On the other hand, the advantage that the adversary wins in $\mathcal{T}_{1}$ Game_Final is negligible. We get the leakage bound by Lemma 1 . Thus, we can obtain the security of the scheme.

A bit more precisely, we use the following table 2 to show the difference of advantages that the adversary wins in the successive two games. Thus, we are easy to obtain the security. Here, we only use the results of Lemma 1 to Lemma 9. The specific proofs of Lemma 1 to Lemma 9 are given in the following. Let $A d v_{\mathcal{A}_{1}}^{\mathcal{T}_{1} \text { Game }{ }_{-}{ }^{k}}\left(\lambda_{d k}, \lambda_{\text {msk }}\right)$ denote the advantage that the adversary $\mathcal{A}_{1}$ wins in game $\mathcal{T}_{1}$ Game $_{-} k(k \in(1, \ldots, Q))$. Let $A d v_{\mathcal{A}_{1}}^{\mathcal{T}_{1} \text { Game }}{ }_{-}^{M s k}\left(\lambda_{d k}, \lambda_{\text {msk }}\right)$ denote the advantage that the adversary $\mathcal{A}_{1}$ wins in game $\mathcal{T}_{1}$ Game $e_{-}$Msk. Let $A d v_{\mathcal{A}_{1}}^{\mathcal{T}_{1} \text { Game }}$ Final $_{-}\left(\lambda_{d k}, \lambda_{\text {msk }}\right)$ denote the advantage that the adversary $\mathcal{A}_{1}$ wins in game $\mathcal{T}_{1}$ Game_Final .

Table 2. The difference of advantages that the adversary wins in the successive two games.

\begin{tabular}{|c|c|c|}
\hline Two successive games & Difference of advantages & $\begin{array}{l}\text { Related } \\
\text { lemmas }\end{array}$ \\
\hline $\begin{array}{l}\mathcal{T}_{1} \text { Game_R } \\
\mathcal{T}_{1}{ }_{-} \text {Game_0 }\end{array}$ & $\left|A d v_{\mathcal{A}_{1}-\text { Game }_{-}{ }^{R}}^{\mathcal{T}_{1}}\left(\lambda_{d k}, \lambda_{m s k}\right)-A d v_{\mathcal{A}_{1}-\text { Game }_{-}{ }^{0}}^{\mathcal{T}_{1}}\left(\lambda_{d k}, \lambda_{m s k}\right)\right| \leq \varepsilon$ & Lemma 2 \\
\hline $\begin{array}{l}\mathcal{T}_{1}{ }_{-} \text {Game_k } \\
\mathcal{T}_{1} \text { Game_k+1 } \\
k \in(0,1, \ldots, Q-1)\end{array}$ & $\left|A d v_{\mathcal{A}_{1}-\text { Game }_{-}{ }^{k}}^{\mathcal{T}_{1}}\left(\lambda_{d k}, \lambda_{m s k}\right)-A d v_{\mathcal{A}_{1}}^{\mathcal{T}_{1} \text { Game }{ }_{-}{ }^{k+1}}\left(\lambda_{d k}, \lambda_{m s k}\right)\right| \leq \varepsilon$ & $\begin{array}{l}\text { Lemma } 3 \\
\text { Lemma } 4 \\
\text { Lemma } 5 \\
\text { Lemma } 6 \\
\end{array}$ \\
\hline $\begin{array}{l}\mathcal{T}_{1} \text { Game_Q } \\
\mathcal{T}_{1}{ }_{\text {_Game_Msk }}\end{array}$ & 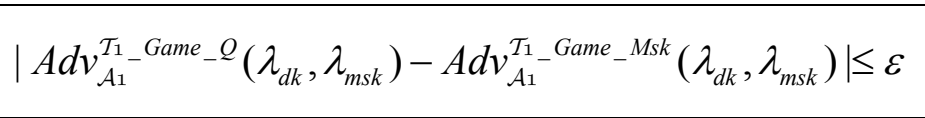 & $\begin{array}{l}\text { Lemma } 7 \\
\text { Lemma } 8\end{array}$ \\
\hline $\begin{array}{l}\mathcal{T}_{1} \text { _Game_Msk and } \\
\mathcal{T}_{1} \text { _Game_Final }\end{array}$ & $\left|A d v_{\mathcal{A}_{1}}^{\mathcal{T}_{1} \text { Game }} Q\left(\lambda_{d k}, \lambda_{m s k}\right)-A d v_{\mathcal{A}_{1}}^{\mathcal{T}_{1_{1} \text { Game }} \text { Final }}\left(\lambda_{d k}, \lambda_{m s k}\right)\right| \leq \varepsilon$ & Lemma 9 \\
\hline
\end{tabular}

From table 2, the security of our scheme is obtained directly. We have:

$$
\begin{aligned}
& \left|A d v_{\mathcal{A}_{1}}^{\mathcal{T}_{1} \text { Game }{ }_{-}{ }^{R}}\left(\lambda_{d k}, \lambda_{m s k}\right)-A d v_{\mathcal{A}_{1}}^{\mathcal{T}_{1} \text { Game_Final }}\left(\lambda_{d k}, \lambda_{m s k}\right)\right| \\
& \mid A d v_{\mathcal{A}_{1}}^{\mathcal{T}_{1} \text { Game }{ }_{-} R}\left(\lambda_{d k}, \lambda_{m s k}\right)-A d v_{\mathcal{A}_{1}}^{\mathcal{T}_{1} \text { Game }_{-}{ }^{0}}\left(\lambda_{d k}, \lambda_{m s k}\right)+A d v_{\mathcal{A}_{1}}^{\mathcal{T}_{1} \text { Game }}{ }^{0}\left(\lambda_{d k}, \lambda_{m s k}\right)-\ldots \\
& =-A d v_{\mathcal{A}_{1}}^{T_{1_{-}} \text {Game }{ }_{-}{ }^{k}}\left(\lambda_{d k}, \lambda_{m s k}\right)+A d v_{\mathcal{A}_{1}}^{\mathcal{T}_{1} \text { Game }{ }_{-}{ }^{k}}\left(\lambda_{d k}, \lambda_{m s k}\right)-\ldots \\
& -A d v_{\mathcal{A}_{1}}^{\mathcal{T}_{1} \text { Game }}{ }^{M s k}\left(\lambda_{d k}, \lambda_{m s k}\right)+A d v_{\mathcal{A}_{1}}^{\mathcal{T}_{1} \text { Game }}{ }^{M s k}\left(\lambda_{d k}, \lambda_{m s k}\right)-A d v_{\mathcal{A}_{1}}^{T_{1_{1}} \text { Game_Final }}\left(\lambda_{d k}, \lambda_{m s k}\right) \mid \\
& \left|A d v_{\mathcal{A}_{1}}^{\mathcal{T}_{1} \text { Game }_{-}{ }^{R}}\left(\lambda_{d k}, \lambda_{m s k}\right)-A d v_{\mathcal{A}_{1}}^{\mathcal{T}_{1_{-}} \text {Game }}{ }_{-}{ }^{0}\left(\lambda_{d k}, \lambda_{m s k}\right)\right| \\
& +\left|A d v_{\mathcal{A}_{1}}^{\mathcal{T}_{1} \text { Game }}{ }^{0}\left(\lambda_{d k}, \lambda_{m s k}\right)-A d v_{\mathcal{A}_{1}}^{\mathcal{T}_{1_{1}} \text { Game }{ }_{-}{ }^{1}}\left(\lambda_{d k}, \lambda_{m s k}\right)\right|+\ldots \\
& \leq+\left|A d v_{\mathcal{A}_{1}}^{\mathcal{T}_{1} \text { Game }{ }_{-}{ }^{k}}\left(\lambda_{d k}, \lambda_{m s k}\right)-A d v_{\mathcal{A}_{1}}^{\mathcal{T}_{1} \text { Game }_{-}{ }^{k+1}}\left(\lambda_{d k}, \lambda_{m s k}\right)\right|+\ldots
\end{aligned}
$$

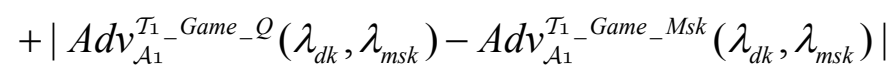

$$
\begin{aligned}
& +\left|A d v_{\mathcal{A}_{1}}^{\tau_{1} \text { Game }}{ }_{-M s k}\left(\lambda_{d k}, \lambda_{m s k}\right)-A d v_{\mathcal{A}_{1}}^{\tau_{1} \text { Game }}{ }^{\text {Final }}\left(\lambda_{d k}, \lambda_{m s k}\right)\right| \\
& \leq \varepsilon+(\mathrm{Q}+1) \varepsilon+\varepsilon=(\mathrm{Q}+3) \varepsilon \text {. }
\end{aligned}
$$

So, $\quad\left|A d v_{\mathcal{A}_{1}}^{\mathcal{T}_{1_{-} \text {Game }}{ }^{R}}\left(\lambda_{d k}, \lambda_{m s k}\right)-A d v_{\mathcal{A}_{1}}^{\mathcal{T}_{1} \text { Game }{ }_{-} \text {Final }}\left(\lambda_{d k}, \lambda_{m s k}\right)\right| \leq(Q+3) \varepsilon \quad$. What's more, $A d v_{\mathcal{A}_{1}}^{\mathcal{T}_{1} \text { Game_Final }}\left(\lambda_{d k}, \lambda_{m s k}\right) \leq \varepsilon$ is proved similarly to that of Theorem 6.8 in the full version of [26]. To sum up, $A d v_{\mathcal{A}_{1}}^{\mathcal{T}_{1} \text { Game }{ }_{-} R}\left(\lambda_{d k}, \lambda_{m s k}\right) \leq \varepsilon$. In addition, Lemma 1 proves the leakage bound. Thus, we finish the proof of Theorem 1.

The specific proofs of Lemma 1 to Lemma 9 are given as follows.

Lemma 1. The leakage size is at most $\lambda_{d k}=\lambda_{\text {msk }}=(n-2 c-1) \lambda$.

Proof. We first introduce a useful conclusion (we called it as Conclusion 1) given in [33]. 
Conclusion 1. Let $p$ be a prime. Let $m \geq l \geq 2(m, l \in N)$. Let $X \leftarrow Z_{p}^{m \times l}, T \leftarrow R k_{1}\left(Z_{p}^{l \times 1}\right)$, $U \leftarrow Z_{p}^{m} . R k_{1}\left(Z_{p}^{l \times 1}\right)$ denotes that the rank of $Z_{p}^{l \times 1}$ is 1 . For an arbitrary leakage function $f: Z_{p}^{m} \rightarrow W \quad, \quad$ if $\quad|W| \leq 4 \cdot\left(1-\frac{1}{p}\right) \cdot p^{l-1} \cdot \varepsilon^{2} \quad$ we $\quad$ have $\quad$ the statistical distance $S D((X, f(X \cdot T)),(X, f(U)) \leq \varepsilon$ where $\varepsilon$ is a negligible value.

Conclusion 1 shows that random subspace is resilient to leakage in the view of information theory. $X \in Z_{p}^{m \times l}$ is a random matrix with rank $l \geq 2 . X$ is also used to denote the linear subspace of dimention $l$ which is generated by columns of this matrix. The function $f$ is an arbitrary leakage function over space $Z_{p}^{m}$. We can see that if the output length of the leakage function is sufficiently short, the random variables $(X, f(V))$ and $(X, f(U))$ are statistically close or indistinguishable, where $V=X \cdot T \subseteq X$ and $U \in Z_{p}^{m}$. Because $f(U)$ and $X$ are independent, $f(U)$ does not leak the information of $X$. Thus, we conclude that if the output length of the leakage function is sufficiently short $f(V)$ does not leak the information of $X$.

We can see $|V|=l \log p$ easily. If the leakage size is at most $\log |W| \leq(l-1) \log p+2 \log \varepsilon$, the leakage $f(V)$ hides the subspace $X$.

In our paper, we use the following Corollary 1.

Corollary 1. Let $p$ be a prime. Let $\vec{\delta} \leftarrow Z_{p}^{m}, \vec{\tau} \leftarrow Z_{p}^{m}, \vec{\tau}^{\prime} \leftarrow Z_{p}^{m}$ such that $\vec{\tau}^{\prime}$ is orthogonal to $\vec{\delta}$ modulo $p$ under the dot product, where $m \geq 3 \quad(m \in N)$. For an arbitrary leakage function $f: Z_{p}^{m} \rightarrow W$, if $|W| \leq 4 \cdot\left(1-\frac{1}{p}\right) \cdot p^{n-1} \cdot \varepsilon^{2}$ we have $S D\left(\left(\vec{\delta}, f\left(\vec{\tau}^{\prime}\right)\right),(\vec{\delta}, f(\vec{\tau}))\right) \leq \varepsilon$ where $\varepsilon$ is a negligible value.

Proof. We apply Conclusion 1 with $l=m-1$. Then, $\vec{\tau}$ corresponds to $U$ and the basis of the orthogonal space of $\vec{\delta}$ corresponds to $X$. We will see that $\vec{\tau}^{\prime}$ is distributed as $X \cdot T$ where $T \leftarrow R k_{1}\left(Z_{p}^{(m-1) \times 1}\right)$. Because $\vec{\delta} \in Z_{p}^{m}$ is selected uniformly at random, $X \leftarrow Z_{p}^{m \times(m-1)}$ is determined by $\vec{\delta}$. Thus, we have $S D\left(\left(\vec{\delta}, f\left(\vec{\tau}^{\prime}\right)\right),(\vec{\delta}, f(\vec{\tau}))\right)=\operatorname{dist}((X, f(X \cdot T)),(X, f(U))$.

If we let $n+1=m, p_{2}=p$ and $\varepsilon=p_{2}^{-c}$, we get that the leakage size is at most $\log |W| \leq$ $(n-1) \log p_{2}-2 c \log p_{2}=(n-2 c-1) \log p_{2}=(n-2 c-1) \lambda$, where $\log p_{2}=\lambda$. Thus, we get that the leakage size is at most $\lambda_{d k}=\lambda_{m s k}=(n-2 c-1) \lambda$.

Lemma 2. Under assumption 1 , the advantage that PPT adversary $\mathcal{A}_{1}$ succeeds in distinguishing $\mathcal{T}_{1}$ Game $R$ and $R$ T $\mathcal{T}_{-}$Game_ 0 is negligible. That is $\left|A d v_{\mathcal{A}_{1}}^{\mathcal{T}_{1} \text { Game }_{-}{ }^{R}}\left(\lambda_{d k}, \lambda_{m s k}\right)-A d v_{\mathcal{A}_{1}}^{\mathcal{T}_{1_{-}} \text {Game }{ }_{-}{ }^{0}}\left(\lambda_{d k}, \bar{\lambda}_{m s k}\right)\right| \leq \varepsilon$.

Proof. If there is a PPT adversary $\mathcal{A}_{1}$ who distinguishes $\mathcal{T}_{1}$ Game $R$ and $\mathcal{T}_{1}$ Game_o with non-negligible advantage, we can construct a simulator $\mathcal{B}$ to break assumption 1.

Firstly, $\mathcal{B}$ is given an instance $\left(N, G, G_{T}, e, g_{1}, g_{3}\right)$ and a challenge item $T$, where $T=g_{1}^{z}$ or $T=g_{1}^{z} g_{2}^{v}$ for $z, v \in Z_{N}$. B interacts with $\mathcal{A}_{1}$ as follows.

Setup Phase: $\mathcal{B}$ firstly creates composite order bilinear groups $\left(N=p_{1} p_{2} p_{3}, G, G_{T}, e\right)$. Then, $\mathcal{B}$ selects $a, b, d \in Z_{N}$ uniformly at random and sets $u_{1}=g_{1}^{a}, h_{1}=g_{1}^{b}$ and $v_{1}=g_{1}^{d}$. Given $g_{1} \in G_{p_{1}}$ and $g_{3} \in G_{p_{3}}, \mathcal{B}$ runs the algorithm Gen of $\Pi$ to generate the common reference string crs and selects random $\left(\alpha, x_{1}, x_{2}, \ldots, x_{n}, r, y_{1}, y_{2}, \ldots, y_{n}\right) \in Z_{N}^{2 n+2}$ and a vector $\vec{\rho}=<\rho_{1}, \rho_{2}, \ldots, \rho_{n+3}>\in Z_{N}^{n+3}$ where $n \geq 2$ is an integer. 
It outputs the master public keympk $=\left(N, G, G_{T}, e, e\left(g_{1}, v_{1}\right)^{\alpha}, g_{1}, g_{1}^{x_{1}}, \ldots, g_{1}^{x_{n}}, u_{1}, h_{1}, v_{1}, g_{3}, c r s\right)$ and the master secret key $m s k=\left(\vec{K}, K_{1}, K_{2}, K_{3}\right)=\left(\left\langle v_{1}^{y_{1}}, \ldots, v_{1}^{y_{n}}\right\rangle, g_{1}^{\alpha} h_{1}^{-r} \prod_{i=1}^{n} g_{1}^{-x_{i} y_{i}}, v_{1}^{r}, u_{1}^{r}\right) * g_{3}^{\vec{\rho}}$.

Oracle Query: For the public key which is not replaced, $\mathcal{B}$ knows the decryption key because he has the $m s k$. Hence, $\mathcal{B}$ can answer the adversary's queries. In addition, $\mathcal{B}$ stores the private key of each user.

Challenge: $\mathcal{A} 1$ gives challenge identity $I D^{*}$ and two message $M_{0}$ and $M_{1}$ to $\mathcal{B} . \mathcal{B}$ scans list $\mathcal{L}_{1}$ to find public key $(Y, \pi)$ of $I D^{*}$ where $Y=e\left(g_{1}, v_{1}\right)^{\alpha \beta}$. If the $\mathcal{A}_{1}$ doesn't replace the public key $\mathcal{B}$ knows $\beta$. Otherwise, $\mathcal{B}$ extracts $\beta$ from $\pi$ by NIZK proof. $\mathcal{B}$ selects $\xi \in\{0,1\}$ at random and outputs the ciphertext: $C^{*}=\left(C_{0}^{*}, \vec{C}^{*}, C_{1}^{*}, C_{2}^{*}\right)=\left(M_{\xi} \cdot e\left(v_{1}^{\beta}, T\right),<T^{x_{1}}, \ldots, T^{x_{n}}>, T^{d}\right.$, $\left.T^{a I D^{*}+b}\right)$.

When $T=g_{1}^{z}$ (that is, no $G_{p_{2}}$ part is contained in $T$ ), the given ciphertext is normal. Thus, $\mathcal{B}$ simulates the $\mathcal{T}_{1}$ Game $R$ correctly.

When $T=g_{1}^{z} g_{2}^{v}$, we set $\vec{\delta}=<x_{1}, \ldots, x_{n}>, \delta_{1}=d, \delta_{2}=a I D^{*}+b$. We can see that $\vec{\delta}, \delta_{1}$ and $\delta_{2}$ are distributed uniformly at random from the adversary's view. The reason is that the $x_{1}, \ldots, x_{n}, d, a$ and $b$ are merely calculated under modulo $p_{1}$ in $m p k$. From the adversary's point of view, $x_{1}, \ldots, x_{n}, d, a$ and $b$ are random under modulo $p_{2}$. Thus, $\mathcal{B}$ simulates the $\mathcal{T}_{1}$ Game_0 correctly.

If $\mathcal{A} 1$ can distinguish $\mathcal{T}_{1}$ Game $R$ and $\mathcal{T}_{1}$ Game_o with non-negligible advantage, $\mathcal{B}$ may break assumption 1 by $\mathcal{A}_{1}$ with non-negligible advantage. Namely, $\left|A d v_{\mathcal{A}_{1}}^{\mathcal{T}_{1} \text { Game }_{-}{ }^{R}}\left(\lambda_{d k}, \lambda_{m s k}\right)-A d v_{\mathcal{A}_{1}}^{\mathcal{T}_{1} \text { Game }_{-}{ }^{0}}\left(\lambda_{d k}, \lambda_{m s k}\right)\right| \leq \varepsilon$.

Lemma 3. If $\lambda_{d k}=(n-2 c-1) \lambda$ and assumption 2 holds, the advantage that PPT adversary $\mathcal{A}_{1}$ distinguishes $\mathcal{T}_{1}$ Game $k$ and $\mathcal{T}_{1}$ Game_k+1 is negligible. That is $\left|A d v_{\mathcal{A}_{1}}^{\mathcal{T}_{1} \text { Game }{ }_{-}{ }^{k}}\left(\lambda_{d k}, \lambda_{m s k}\right)-A d v_{\mathcal{A}_{1}}^{\mathcal{T}_{1_{-}} \text {Game }{ }_{-}{ }^{k+1}}\left(\lambda_{d k}, \lambda_{m s k}\right)\right| \leq \varepsilon$.

Proof. In order to prove the Lemma 3 , another game which is called $\mathcal{T}_{1}$ AltGame $k$ is defined. Compared with $\mathcal{T}_{1}$ Game_k, in $\mathcal{T}_{1}$ AltGame_ $k$ the certificate is normal instead of semi-functional if the $\bar{k}^{\text {th }}$ query are create oracle. The decryption key is still semi-functional. Therefore, the Lemma 3 can be proved by the following three lemmas.

Lemma 4. If $\lambda_{d k}=(n-2 c-1) \lambda$ and assumption 2 holds, the advantage that PPT adversary $\mathcal{A}_{1}$ can succeed in distinguishing $\mathcal{T}_{1}$ Game $k$ and $\mathcal{T}_{1_{-}}$AltGame $k_{-}$is negligible. That is $\left|A d v_{\mathcal{A}_{1}}^{\mathcal{T}_{1} \text { Game }_{-}{ }^{k}}\left(\lambda_{d k}, \lambda_{m s k}\right)-A d v_{\mathcal{A}_{1}}^{\mathcal{T}_{1} \text { AltGame }_{-}{ }^{k}}\left(\lambda_{d k}, \lambda_{\text {msk }}\right)\right| \leq \varepsilon$.

Compared with $\mathcal{T}_{1}$ Game $k$, in $\mathcal{T}_{1}$ AltGame_ $k$ the certificate is normal instead of semi-functional if the $k^{t h}$ query are create oracle. The decryption key is still semi-functional.

Proof. If there is a PPT adversary $\mathcal{A}_{1}$ who distinguishes $\mathcal{T}_{1}$ Game $k$ and $\mathcal{T}_{1}$ AltGame $k$ with non-negligible advantage, we can construct a simulator $\mathcal{B}$ who can break assumption 2. Firstly, $\mathcal{B}$ is given an instance $\left(N, G, G_{T}, e, g_{1}, g_{3}, g_{1}^{z} g_{2}^{v}, g_{2}^{u} g_{3}^{\rho}\right)$ and a challenge item $T$ which is $g_{1}^{\omega} g_{3}^{\sigma}$ or $g_{1}^{\omega} g_{2}^{\kappa} g_{3}^{\sigma}$. $\mathcal{B}$ interacts with $\mathcal{A}_{1}$ as follows.

Setup Phase: $\mathcal{B}$ firstly creates composite order bilinear groups $\left(N=p_{1} p_{2} p_{3}, G, G_{T}, e\right) . \mathcal{B}$ selects $a, b, d \in Z_{N}$ uniformly at random and sets $u_{1}=g_{1}^{a}, \quad h_{1}=g_{1}^{b}$ and $v_{1}=g_{1}^{d}$. Given $g_{1} \in G_{p_{1}}$ and $g_{3} \in G_{p_{3}}, \mathcal{B}$ runs the algorithm Gen of $\Pi$ to generate the common reference 
string crs and selects random $\left(\alpha, x_{1}, x_{2}, \ldots, x_{n}, r, y_{1}, y_{2}, \ldots, y_{n}\right) \in Z_{N}^{2 n+2}$ and a vector $\vec{\rho}=<\rho_{1}, \rho_{2}, \ldots, \rho_{n+3}>\in Z_{N}^{n+3}$ where $n \geq 2$ is an integer.

It outputs the master public keympk $=\left(N, G, G_{T}, e, e\left(g_{1}, v_{1}\right)^{\alpha}, g_{1}, g_{1}^{x_{1}}, \ldots, g_{1}^{x_{n}}, u_{1}, h_{1}, v_{1}, g_{3}\right.$, crs $)$ and the master secret key $m s k=\left(\vec{K}, K_{1}, K_{2}, K_{3}\right)=\left(\left\langle v_{1}^{y_{1}}, \ldots, v_{1}^{y_{n}}\right\rangle, g_{1}^{\alpha} h_{1}^{-r} \prod_{i=1}^{n} g_{1}^{-x_{i} y_{i}}, v_{1}^{r}, u_{1}^{r}\right) * g_{3}^{\vec{\rho}}$.

Oracle Query: $\mathcal{B}$ may reply every query because he knows $\alpha, x_{i}, y_{i}, r$. Specifically, $\mathcal{B}$ answers the $j^{\text {th }}$ query for $I D_{j}$ as follows.

(1) If it is the create query, $\mathcal{B}$ generates normal certificate $\operatorname{Cert}_{I D_{j}}$ by using $m s k$. The $\operatorname{Cert}_{I D_{j}}$ can leak information. $\mathcal{B}$ runs SetPrivateKey to get $\beta$ and runs SetPublicKey to get $p k_{I D_{j}} . \quad \mathcal{B}$ picks randomly $\left(\omega_{1}, \omega_{2}, \ldots, \omega_{n}, t\right) \in Z_{N}^{n+1}$ and $\vec{\rho} \in Z_{N}^{n+2}$.

(a) If $j \leq k, \mathcal{B}$ selects randomly a vector $\vec{\gamma} \in Z_{N}^{n+2}$ and calculates the decryption key $d k_{I D_{j}}$ $=\left(<v_{1}^{\omega_{1}}, \ldots, v_{1}^{\omega_{n}}>, g^{\alpha \beta}\left(u_{1}^{I D_{j}} h_{1}\right)^{-t} \prod_{i=1}^{n} g_{1}^{-x_{i} \omega_{i}}, v_{1}^{t}\right) *\left(g_{2}^{\mu} g_{3}^{\rho}\right)^{\vec{\gamma}} * g_{3}^{\vec{\rho}}$.

(b) If $j>k+1, \mathcal{B}$ calculates the decryption key $d k_{I D_{j}}=\left(<v_{1}^{\omega_{1}}, \ldots, v_{1}^{\omega_{n}}\right\rangle, g^{\alpha \beta}\left(u_{1}^{I D_{j}} h_{1}\right)^{-t}$ $\left.\prod_{i=1}^{n} g_{1}^{-x_{i} \omega_{i}}, v_{1}^{t}\right) * g_{3}^{\vec{\rho}}$

(c) If $j=k+1, \mathcal{B}$ calculates the decryption key $d k_{I D_{k+1}}=\left(<T^{d w_{1}}, \ldots, T^{d w_{n}}\right\rangle, T^{-\left(a I D_{k+1}+b\right)} *$ $\left.\prod_{i=1}^{n} T^{-x_{i} \omega_{i}} * g_{1}^{\alpha \beta}, T^{d}\right) * g_{3}^{\vec{\rho}}$ by using the challenge item $T$.

If $T=g_{1}^{\omega} g_{3}^{\sigma}$, from the $\mathcal{B}$ 's point of view, the decryption key is distributed uniformly at random because no $G_{p_{2}}$ part is in $T$.

If $T=g_{1}^{\omega} g_{2}^{\kappa} g_{3}^{\sigma}$, the decryption key is semi-functional and the corresponding parameters are: $\vec{\theta}=<\kappa d w_{1}, \ldots, \kappa d w_{n}>, \theta_{1}=-\kappa\left(a I D_{k+1}+b+\sum_{i=1}^{n} x_{i} w_{i}\right), \theta_{2}=\kappa d$.

Because $w_{1}, \ldots, w_{n}, x_{1}, \ldots, x_{n}, d, a, b$ are merely calculated by modulo $p_{1}$ in $m p k$, from the $\mathcal{A}_{1}$ 's point of view, they are random by modulo $p_{2}$. Thus, the semi-functional decryption key is properly distributed.

$\mathcal{B}$ sets $H \leftarrow H+1$, adds the item $\left(H, I D_{j}, I D_{j}^{\prime}, p k_{I D_{j}}, s k_{I D_{j}} \operatorname{Cert}_{I D_{j}}, d k_{I D_{j}}, 0,0\right)$ to list $\mathcal{L}_{1}$ and returns $H$ to $\mathcal{A}_{1}$.

(2) If it is the replace public key query with $p k_{I D_{j}}^{\prime}, \mathcal{B}$ picks randomly $\left(z_{1}, z_{2}, \ldots, z_{n}, r\right) \in Z_{N}^{n+1}$ and $\vec{\rho} \in Z_{N}^{n+2}$.

(a) If $j \leq k, \mathcal{B}$ randomly selects a vector $\vec{\gamma} \in Z_{N}^{n+2}$ and calculates the certificate $\operatorname{Cert}_{I D_{j}}$ $=\left(<v_{1}^{z_{1}}, \ldots, v_{1}^{z_{n}}>, g_{1}^{\alpha}\left(u_{1}^{I D_{j}} h_{1}\right)^{-r} \prod_{i=1}^{n} g_{1}^{-x_{i} z_{i}}, v_{1}^{r}\right) *\left(g_{2}^{\mu} g_{3}^{\rho}\right)^{\vec{\gamma}} * g_{3}^{\vec{\rho}}$.

(b) If $j>k+1, \mathcal{B}$ calculates the certificate $\operatorname{Cert}_{I D_{j}}=\left(<v_{1}^{z_{1}}, \ldots, v_{1}^{z_{n}}>, g_{1}^{\alpha}\left(u_{1}^{I D_{j}} h_{1}\right)^{-t} \prod_{i=1}^{n} g_{1}^{-x_{i} z_{i}}\right.$, $\left.v_{1}^{r}\right) * g_{3}^{\vec{\rho}}$.

(c) If $j=k+1$, by using the challenge item $T$ the challenger $\mathcal{B}$ calculates the certificate 
$\operatorname{Cert}_{I D_{k+1}}=\left(<T^{d z_{1}}, \ldots, T^{d z_{n}}>, T^{-\left(a I D_{k+1}+b\right)} \prod_{i=1}^{n} T^{-x_{i} z_{i}} * g_{1}^{\alpha}, T^{d}\right) * g_{3}^{\vec{\rho}}$.

If $T=g_{1}^{\omega} g_{3}^{\sigma}$, from the $\mathcal{B}$ 's point of view, the certificate is properly distributed because no $G_{p_{2}}$ part is in $T$.

If $T=g_{1}^{\omega} g_{2}^{\kappa} g_{3}^{\sigma}$, the certificate is semi-functional and we set $\vec{\eta}=<\kappa d z_{1}, \ldots, \kappa d z_{n}>, \eta_{1}=-\kappa($ $\left.a I D_{k+1}+b+\sum_{i=1}^{n} x_{i} z_{i}\right)$ and $\eta_{2}=\kappa d$.

Because $z_{1}, \ldots, \mathrm{z}_{n}, x_{1}, \ldots, x_{n}, d, a, b$ are merely calculated by modulo $p_{1}$ in $m p k$, from the $\mathcal{A}_{1}$ 's point of view, they are random by modulo $p_{2}$. Thus, the SF certificate is properly distributed.

$\mathcal{B}$ sets $H \leftarrow H+1$, adds the item $\left(H, I D_{j}, I D_{j}^{\prime}, p k_{I D_{j}}^{\prime}, \perp, \operatorname{Cert}_{I D_{j}}, \perp, 0,0\right)$ to list $\mathcal{L} 1$, and returns $H$ to $\mathcal{A}_{1}$.

Challenge: $\mathcal{A}_{1}$ gives an identity $I D^{*}$ and two messages $M_{0}$ and $M_{1} . \mathcal{B}$ selects a random bit $\xi \in\{0,1\} . \mathcal{B}$ scans the list $\mathcal{L}_{1}$ to find $p k_{I D_{j}}=(Y, \pi)$ about $I D^{*}$ for the largest handle where $Y=e\left(g_{1}, v_{1}\right)^{\alpha \beta^{*}}$. If the $\mathcal{A} 1$ doesn't replace the public key $\mathcal{B}$ knows $\beta^{*}$. Otherwise, $\mathcal{B}$ can extract $\beta^{*}$ from $\pi$ by NIZK proof. By using $g_{1}^{z} g_{2}^{v} \mathcal{B}$ outputs the SF ciphertext $C^{*}=\left(C_{0}^{*}, \vec{C}^{*}, C_{1}^{*}, C_{2}^{*}\right)=\left(M_{\xi} \cdot e\left(v_{1}^{\beta^{*}}, g_{1}^{z} g_{2}^{v}\right)^{\alpha},<\left(g_{1}^{z} g_{2}^{v}\right)^{x_{1}}, \cdots,\left(g_{1}^{z} g_{2}^{v}\right)^{x_{n}}>,\left(g_{1}^{z} g_{2}^{v}\right)^{d},\left(g_{1}^{z} g_{2}^{v}\right)^{a I D^{*}+b}\right)$.

From the SF ciphertext we get SF parameters $\vec{\delta}=<v x_{1}, \ldots, v x_{n}>, \delta_{1}=v d, \delta_{2}=v\left(a I D^{*}+b\right)$. For the same reason as before, from the $\mathcal{A}_{1}$ 's point of view, $x_{1}, \ldots, x_{n}, d, a, b$ are random under modulo $p_{2}$ and the given ciphertext is distributed uniformly at random if $I D^{*}$ isn't the identity for the $(k+1)^{t h}$ handle.

( I ) If the $(k+1)^{t h}$ inquiry is create inquiry, we can get the conclusion: If $T$ contains $G_{p_{2}}$ part, the decryption key towards the $(k+1)^{t h}$ handle is semi-functional for the $C^{*}$. The NSF property is embodied as follows. $\vec{\theta} \cdot \vec{\delta}=\kappa v d \sum_{i=1}^{n} x_{i} w_{i} \bmod p_{2}, \quad \theta_{1} \cdot \delta_{1}=-\kappa v d\left(a I D_{k+1}+b+\sum_{i=1}^{n} x_{i} w_{i}\right)$ $\bmod p_{2}$ and $\theta_{2} \cdot \delta_{2}=-\kappa v d\left(a I D^{*}+b\right) \bmod p_{2}$.

If $T=g_{1}^{w} g_{2}^{\kappa} g_{3}^{\sigma}, \mathcal{B}$ imitates the $\mathcal{T}_{1}{ }_{-}$AltGame ${ }_{-} k$ correctly when $I D^{*} \neq I D_{k+1} \bmod p_{2}$. Or else, $\mathcal{B}$ imitates the $\mathcal{T}_{1}$ Game_k.

(II) If the $(k+1)^{\text {th }}$ inquiry is $\mathcal{O}$-Replacepublickey, we can get the conclusion: Though the public key is replaced, the decryption key has the same $G_{p_{2}}$ part with the corresponding certificate. The reason is that if $\mathcal{A}_{1}$ doesn't affect the $G_{p_{1}}$ part $\mathcal{A}_{1}$ can't randomize $G_{p_{2}}$ part. The NSF property is embodied as follows.

$\vec{\theta} \cdot \vec{\delta}=\vec{\eta}^{\beta} \cdot \vec{\delta}=\kappa v \beta d \sum_{i=1}^{n} x_{i} w_{i} \bmod p_{2}, \theta_{1} \cdot \delta_{1}=\eta_{1}^{\beta} \cdot \delta_{1}=-\kappa v \beta d\left(a I D_{k+1}+b+\sum_{i=1}^{n} x_{i} w_{i}\right) \bmod p_{2}$ and $\theta_{2} \cdot \delta_{2}=\eta_{2}^{\beta} \cdot \delta_{2}=-\kappa v \beta d\left(a I D^{*}+b\right) \bmod p_{2}$.

If $T=g_{1}^{w} g_{2}^{\kappa} g_{3}^{\sigma} \mathcal{B}$ imitates the $\mathcal{T}_{1}{ }_{-}$AltGame_k correctly when $I D^{*} \neq I D_{k+1} \bmod p_{2}$. Or else, $\mathcal{B}$ imitates the $\mathcal{T}_{1}$ Game $k$.

In the above two cases, NSF is taken into account as follows.

(1) $I D^{*}=I D_{k+1} \bmod p_{2}$ and $I D^{*} \neq I D_{k+1} \bmod N$.

(2) $I D^{*}=I D_{k+1} \bmod N$. 
For the case (1), if $\mathcal{B}$ computes $a^{\prime}=\operatorname{gcd}\left(I D^{*}-I D_{k+1}, N\right)$ he can generate a nontrivial factor of $N$. Subgroup decision assumption is broken, which implies that assumption 1, 2, 3 are broken.

For the case (2), the challenge identity $I D^{*}$ is pointed out by the $(k+1)^{\text {th }}$ handle. In the case, $\mathcal{A} 1$ can't extract the decryption key of $I D^{*}$. We further divide it into two subcases:

(a) $\mathcal{A}_{1}$ may obtain the certificate and some leakage of the decryption key for identity $I D^{*}$.

(b) $\mathcal{A}_{1}$ is able to replace the corresponding public key and get some leakage of the certificate for identity $I D^{*}$.

The following Lemma 5 is to show that normal SF and NSF are indistinguishable.

Lemma 5. For the case (2) of Lemma 4, when the decryption key/certificate changes from normal SF to NSF for the $(k+1)^{\text {th }}$ handle of identity $I D^{*}$ in the case of $\lambda_{d k}$ leakage of the decryption key, the advantage that $\mathcal{A}_{1}$ wins is a negligible value, where $\lambda_{d k}=(n-2 c-1) \lambda$ and $c$ is a fixed positive constant.

Proof. If there is a PPT adversary $\mathcal{A}_{1}$ who distinguishes the SF and NSF decryption key or certificate with non-negligible advantage, we can construct an algorithm $\mathcal{B}$ to break Corollary 1 . That is to say, the advantage that $\mathcal{B}$ succeeds in distinguishing the distribution $\left(\vec{\delta}, f\left(\vec{\tau}^{\prime}\right)\right)$ and $(\vec{\delta}, f(\vec{\tau}))$ is non-negligible. Thus, there is a contradiction.

$\mathcal{B}$ runs Setup. $\mathcal{B}$ keeps the $m s k$ and gives the $m p k$ to $\mathcal{A}_{1}$. Because $\mathcal{B}$ has the $m s k$ and knows $g_{2} \in G_{p_{2}}$, he can generate normal and semi-functional keys. Thus, he can answer all the inquiries of $\mathcal{A}_{1}$.

Towards the $(k+1)^{t h}$ replace public key or create inquiry about identity $I D, \mathcal{B}$ replies with the handle $H^{*}$ and doesn't create the private key. If $\mathcal{A}_{1}$ asks for the leakage of decryption key for $\left(H^{*}, f\right), \mathcal{B}$ encodes the leakage that $\mathcal{A}_{1}$ inquiries in Phase 1 for the $I D$ as a PPT function $f$ : $Z_{p_{2}}^{n} \rightarrow 2^{\lambda_{d k}}$. This can be done if all the values of other keys and other variables for the challenge key are regarded as fixed. Then $\mathcal{B}$ gets $(\vec{\delta}, f(\vec{\Gamma}))$, where $\vec{\Gamma}$ is $\vec{\tau}$ or $\vec{\tau}^{\prime}$ according to Corollary 1 and has $n+1$ components. $\mathcal{B}$ returns $f(\vec{\Gamma})$ to $\mathcal{A}_{1}$ as the leakage about the $(k+1)^{\text {th }}$ handle, which defines the challenge keys in the following.

(1) If the $(k+1)^{t h}$ inquiry is create query, $\mathcal{B}$ calculates normal certificate $\operatorname{Cert}_{I D}$ by using msk. $\mathcal{B}$ selects randomly $r_{1}, r_{2} \in Z_{p_{2}}$ and sets implicitly $G_{p_{2}}$ part in decryption key to be $g_{2}^{\vec{\Gamma}^{\prime}}$, where $\overrightarrow{\Gamma^{\prime}}$ is defined as $<\vec{\Gamma}, 0>+<\underbrace{0, \ldots, 0, r_{1}}_{n}, r_{2}>$. B sets non- $G_{p_{2}}$ part in decryption key to satisfy the proper distribution. It is able to solve the subcase (a) of Lemma 4.

(2) If the $(k+1)^{\text {th }}$ inquiry is $\mathcal{O}$-Replacepublickey, $\mathcal{B}$ selects randomly $r_{1}, r_{2} \in Z_{p_{2}}$ and sets

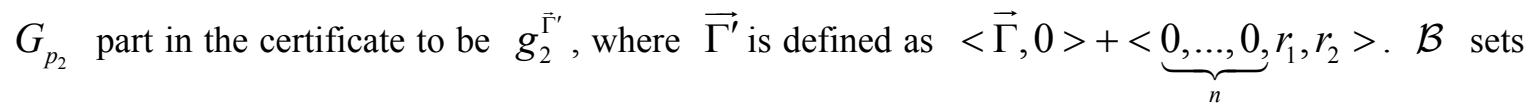
non- $G_{p_{2}}$ part in the certificate to satisfy the proper distribution. It is able to solve the subcase (b) of Lemma 4.

In certain circumstances, $\mathcal{A}_{1}$ gives the challenge message. $\mathcal{B}$ selects $t_{2} \in Z_{p_{2}}$ which satisfies $\delta_{n} r_{1}+t_{2} r_{2} \equiv 0 \bmod p_{2}$. By using $<\vec{\delta}, 0>+<0, \ldots, 0,0, t_{2}>$ as $G_{p_{2}}$ part where $\vec{\delta}$ has $n+1$ components, $\mathcal{B}$ generates the challenge ciphertext. If $\vec{\delta}$ and $\vec{\Gamma}$ are orthogonal, the $(k+1)^{\text {th }}$ handle is about the NSF decryption key/certificate.

It is obvious that $\mathcal{B}$ is able to reply easily the inquiries in Phase 2 . By using the output of $\mathcal{A}_{1}$, 
$\mathcal{B}$ succeeds in distinguishing the distribution $\left(\vec{\delta}, f\left(\vec{\tau}^{\prime}\right)\right)$ and $(\vec{\delta}, f(\vec{\tau}))$ with non-negligible advantage.

If assumption 2 holds the $\mathcal{T}_{1}$ Game $k$ and $\mathcal{T}_{1}$ AltGame $k_{-}$are indistinguishable from the adversary's point of view. Namely, $\left|A d v_{\mathcal{A}_{1}}^{\mathcal{T}_{1} \text { Game }{ }^{k}}\left(\lambda_{d k}, \lambda_{m s k}\right)-A d v_{\mathcal{A}_{1}}^{\mathcal{T}_{1} \text { AltGame }{ }_{-}{ }^{k}}\left(\lambda_{d k}, \lambda_{m s k}\right)\right| \leq \varepsilon$.

Lemma 6. If $\lambda_{d k}=(n-2 c-1) \lambda$ and assumption 2 holds, $\mathcal{T}_{1}$ AltGame_$_{-} k$ and $\mathcal{T}_{1}$ Game_ $k+1$ are indistinguishable from the adversary's point of view. That is $\left|A d v_{\mathcal{A}_{1}}^{\mathcal{T}_{1} \text { AltGame }{ }_{-}{ }^{2}}\left(\lambda_{d k}, \lambda_{m s k}\right)-A d v_{\mathcal{A}_{1}}^{\mathcal{T}_{1_{-}} \text {Game }{ }_{-} k+1}\left(\lambda_{d k}, \lambda_{m s k}\right)\right| \leq \varepsilon$.

Proof. If there is a PPT adversary $\mathcal{A}_{1}$ who distinguishes the $\mathcal{T}_{1}$ AltGame $_{-} k$ and $\mathcal{T}_{1}$ Game_ $k+1$ with non-negligible advantage, we can construct a simulator $\mathcal{B}$ who breaks assumption 2. Firstly, $\mathcal{B}$ is given an instance $\left(N, G, G_{T}, e, g_{1}, g_{3}, g_{1}^{z} g_{2}^{v}, g_{2}^{u} g_{3}^{\rho}\right) . \mathcal{B}$ is given a challenge item $T$ which is $g_{1}^{\omega} g_{3}^{\sigma}$ or $g_{1}^{\omega} g_{2}^{\kappa} g_{3}^{\sigma}$. $\mathcal{B}$ interacts with $\mathcal{A}_{1}$ as follows.

Setup Phase: $\mathcal{B}$ firstly creates composite order bilinear groups $\left(N=p_{1} p_{2} p_{3}, G, G_{T}, e\right) . \mathcal{B}$ selects $a, b, d \in Z_{N}$ uniformly at random and sets $u_{1}=g_{1}^{a}, h_{1}=g_{1}^{b}$ and $v_{1}=g_{1}^{d}$. Given $g_{1} \in G_{p_{1}}$ and $g_{3} \in G_{p_{3}}, \mathcal{B}$ runs the algorithm $G e n$ of $\Pi$ to generate the common reference string crs and selects random $\left(\alpha, x_{1}, x_{2}, \ldots, x_{n}, r, y_{1}, y_{2}, \ldots, y_{n}\right) \quad \in Z_{N}^{2 n+2}$ and a vector $\vec{\rho}=<\rho_{1}, \rho_{2}, \ldots, \rho_{n+3}>\in Z_{N}^{n+3}$ where $n \geq 2$ is an integer.

It outputs the master public key $m p k=\left(N, G, G_{T}, e, e\left(g_{1}, v_{1}\right)^{\alpha}, g_{1}, g_{1}^{x_{1}}, \ldots, g_{1}^{x_{n}}, u_{1}, h_{1}, v_{1}, g_{3}, c r s\right)$ and the master secret key $\left.m s k=\left(<v_{1}^{y_{1}}, \ldots, v_{1}^{y_{n}}\right\rangle, g_{1}^{\alpha} h_{1}^{-r} \prod_{i=1}^{n} g_{1}^{-x_{i} y_{i}}, v_{1}^{r}, u_{1}^{r}\right) * g_{3}^{\vec{\rho}} \triangleq\left(\vec{K}, K_{1}, K_{2}, K_{3}\right)$.

Oracle Query: $\mathcal{B}$ may reply every query because he knows $\alpha, x_{i}, y_{i}, r$. Specifically, $\mathcal{B}$ answers the $j^{\text {th }}$ query for identity $I D_{j}$ as follows.

(1) If it is the create query, $\mathcal{B}$ generates normal certificate $\operatorname{Cert}_{I D_{j}}=\left(\vec{D}, D_{1}, D_{2}\right)$ by using $m s k$. The $\operatorname{Cert}_{I D_{j}}$ can leak information. $\mathcal{B}$ runs SetPrivateKey to get $\beta$ and runs SetPublicKey to get $p k_{I D_{j}}$.

(a) If $j \leq k, \mathcal{B}$ selects randomly vectors $\vec{\rho}_{1}, \vec{\gamma}_{1}, \vec{\rho}_{2}, \vec{\gamma}_{2} \in Z_{N}^{n+2}$ and calculates the decryption key: $\widetilde{\operatorname{Cert}_{I D}}=\left(\vec{D}, D_{1}, D_{2}\right) *\left(g_{2}^{\mu} g_{3}^{\rho}\right)^{\vec{\gamma}_{1}} * g_{3}^{\vec{\rho}_{1}}, \widetilde{d k_{I D}}=\left(\vec{S}, S_{1}, S_{2}\right) *\left(g_{2}^{\mu} g_{3}^{\rho}\right)^{\vec{\gamma}_{2}} * g_{3}^{\vec{\rho}_{2}} . \mathcal{B}$ adds $\left(H, I D_{j}\right.$, $\left.I D_{j}^{\prime}, p k_{I D_{j}}, s k_{I D_{j}}, \overline{\operatorname{Cert}_{I D_{j}}}, \widetilde{d k_{I D_{j}}}, 0,0\right)$ to the list $\mathcal{L}_{1}$.

(b) If $j>k+1, \mathcal{B}$ calculates normally the decryption key $d k_{I D_{j}}$ and adds $\left(H, I D_{j}, I D_{j}^{\prime}, p k_{I D_{j}}\right.$, $\left.s k_{I D_{j}}, \operatorname{Cert}_{I D_{j}}, d k_{I D_{j}}, 0,0\right)$ to the list $\mathcal{L}_{1}$.

(c) If $j=k+1, \mathcal{B}$ selects randomly vectors $\vec{\rho}_{1}, \vec{\rho}_{2}, \vec{\gamma}_{1} \in Z_{N}^{n+2}$ and $\left(z_{1}, z_{2}, \ldots, z_{n}\right) \in Z_{N}^{n}$. By using the challenge item $\mathcal{B}$ calculates the certificate $\operatorname{Cert}_{I D_{k+1}}=\left(\left\langle T^{d_{1} z_{1}}, \ldots, T^{d_{1} z_{n}}\right\rangle, T^{-\left(a I D_{k+1}+b\right)}\right.$ $\left.\prod_{i=1}^{n} T^{-x_{i} z_{i}} * g^{\alpha}, T^{d}\right) * g_{3}^{\vec{\rho}_{1}}$.

$\mathcal{B}$ invokes SetDecryptKey to get decryption key $d k_{I D_{j}}$ and calculates SF decryption key $\widetilde{d k_{I D_{j}}}=\left(\vec{S}, S_{1}, S_{2}\right) *\left(g_{2}^{\mu} g_{3}^{\rho}\right)^{\vec{\gamma}_{2}} * g_{3}^{\vec{\rho}_{2}}$. $\mathcal{B}$ adds $\left(H, I D_{j}, I D_{j}^{\prime}, p k_{I D_{j}}, s k_{I D_{j}}, \operatorname{Cert}_{I D_{j}}, \widetilde{d k_{I D_{j}}}, 0,0\right)$ to the list $\mathcal{L}_{1}$.

If $T=g_{1}^{\omega} g_{3}^{\sigma}$, from the $\mathcal{B}$ 's point of view, the decryption key is uniformly distributed at random 
because no $G_{p_{2}}$ part is in $T$.

If $T=g_{1}^{\omega} g_{2}^{\kappa} g_{3}^{\sigma}$, the decryption key is semi-functional and the corresponding parameters are $\vec{\eta}=<\kappa d_{1} z_{1}, \ldots, \kappa d_{1} z_{n}>, \eta_{1}=-\kappa\left(a I D_{k+1}+b+\sum_{i=1}^{n} x_{i} z_{i}\right)$ and $\eta_{2}=\kappa d$.

Because $z_{1}, \ldots, z_{n}, x_{1}, \ldots, x_{n}, d, a, b$ are merely calculated by modulo $p_{1}$ in $m p k$, from the $\mathcal{A}_{1}$ 's point of view, they are random by modulo $p_{2}$. Thus, the semi-functional decryption key is properly distributed.

$\mathcal{B}$ sets $H \leftarrow H+1$ and returns $H+1$ to $\mathcal{A}_{1}$.

(2) If the $j^{\text {th }}$ inquiry is replace public key inquiry, the simulation is run in the same way as that of Lemma 4.

Challenge: It is run in the same way as the Challenge of Lemma 4.

The NSF property is analyzed in a similar way as that of Lemma 4.

To summarize, if the assumption 2 holds and the leakage amount is at most $(n-2 c-1) \lambda$, $\mathcal{T}_{1}$ Game $k k$ and $\mathcal{T}_{1}$ AltGame $k$ are indistinguishable from the adversary's point of view. Likewise, $\mathcal{T}_{1}$ AltGame $_{k} k$ and $\mathcal{T}_{1}{ }_{-}$Game_ $k+1$ are indistinguishable from the adversary's point of view. Thus, $\mathcal{T}_{1}$ Game ${ }_{-} k$ and $\mathcal{T}_{1}$ Game ${ }_{-} k+1$ are indistinguishable from the adversary's point of view.

By Lemma 4 to Lemma 6, we have

$$
\begin{aligned}
& \left|A d v_{\mathcal{A}_{1}}^{\mathcal{T}_{1} \text { Game }{ }_{-}{ }^{k}}\left(\lambda_{d k}, \lambda_{m s k}\right)-A d v_{\mathcal{A}_{1}}^{\mathcal{T}_{1} \text { Game }{ }^{k+1}}\left(\lambda_{d k}, \lambda_{m s k}\right)\right| \\
& \mid A d v_{\mathcal{A}_{1}}^{\mathcal{T}_{1} \text { Game }{ }_{-} k}\left(\lambda_{d k}, \lambda_{m s k}\right)-A d v_{\mathcal{A}_{1}}^{\mathcal{T}_{1} \text { AltGame }{ }^{k}}\left(\lambda_{d k}, \lambda_{m s k}\right)+A d v_{\mathcal{A}_{1}-{ }_{1}}^{\mathcal{T}_{1} \text { AltGame }{ }^{k}}\left(\lambda_{d k}, \lambda_{m s k}\right) \\
& -A d v_{\mathcal{A}_{1}}^{\mathcal{T}_{1} \text { Game }_{-}{ }^{k+1}}\left(\lambda_{d k}, \lambda_{m s k}\right) \mid \\
& \leq\left|A d v_{\mathcal{A}_{1}}^{\mathcal{T}_{1} \text { Game }{ }^{k}}\left(\lambda_{d k}, \lambda_{m s k}\right)-A d v_{\mathcal{A}_{1}}^{\mathcal{T}_{1_{-}} \text {AltGame }{ }_{-}{ }^{\prime}}\left(\lambda_{d k}, \lambda_{m s k}\right)\right| \\
& +\left|A d v_{\mathcal{A}_{1}}^{\mathcal{T}_{1}{ }^{\text {AltGame }}{ }^{k}}\left(\lambda_{d k}, \lambda_{\text {msk }}\right)-A d v_{\mathcal{A}_{1}}^{\mathcal{T}_{1_{-} \text {Game }}{ }^{k+1}}\left(\lambda_{d k}, \lambda_{\text {msk }}\right)\right|
\end{aligned}
$$

$=\varepsilon+\varepsilon=2 \varepsilon$. This finishes Lemma 3 . Thus, we have

$$
\left|A d v_{\mathcal{A}_{1}}^{\mathcal{T}_{1} \text { Game }{ }_{-} k}\left(\lambda_{d k}, \lambda_{m s k}\right)-A d v_{\mathcal{A}_{1}}^{\mathcal{T}_{1_{-}} \text {Game }{ }_{-}{ }^{k+1}}\left(\lambda_{d k}, \lambda_{m s k}\right)\right| \leq \varepsilon .
$$

Lemma 7. If $\lambda_{m s k}=(n-2 c-1) \lambda$ and assumption 2 holds, $\mathcal{T}_{1}$ Game_$Q$ and $\mathcal{T}_{1}$ Game_Msk are indistinguishable from the adversary's point of view. That is $\left|A d v_{\mathcal{A}_{1}}^{\mathcal{T}_{1} \text { Game } Q_{-}}\left(\lambda_{d k}, \lambda_{m s k}\right)-A d v_{\mathcal{A}_{1}}^{\mathcal{T}_{1} \text { Game_Msk }}\left(\lambda_{d k}, \lambda_{m s k}\right)\right| \leq \varepsilon$.

Proof. If there is a PPT adversary $\mathcal{A}_{1}$ who may distinguish the $\mathcal{T}_{1}$ Game $Q$ and $\mathcal{T}_{1}$ _Game_Msk with non-negligible advantage, we can construct a simulator $\mathcal{B}$ who breaks assumption 2. Firstly, $\mathcal{B}$ is given an instance $\left(N, G, G_{T}, e, g_{1}, g_{3}, g_{1}^{z} g_{2}^{v}, g_{2}^{u} g_{3}^{\rho}\right) . \mathcal{B}$ is given a challenge item $T$ which is $g_{1}^{\omega} g_{3}^{\sigma}$ or $g_{1}^{\omega} g_{2}^{\kappa} g_{3}^{\sigma}$. $\mathcal{B}$ interacts with $\mathcal{A}_{1}$ as follows.

Setup Phase: $\mathcal{B}$ selects $\alpha, a, b, d, x_{1}, \ldots, x_{n} \in Z_{n} \quad$ and $\quad$ sets $g_{1}=g_{1}, u_{1}=g_{1}^{a}, h_{1}=g_{1}^{b} \quad$ and $v_{1}=g^{d} . \mathcal{B}$ issues the $m p k=\left(N, G, G_{T}, e, g_{1}, u_{1}, h_{1}, q_{1}, v_{1}, e\left(g_{1}, v_{1}\right)^{\alpha}, g_{1}^{x_{i}}\right)$. $\mathcal{B}$ selects $\left(y_{1}, \ldots, y_{n}\right) \in Z_{N}^{n}$ and $\vec{\rho} \in Z_{N}^{n+3}$. By using the challenge item $\mathcal{B}$ generates the master secret key $m s k=\left(<T^{d y_{1}}, \ldots, T^{d y_{n}}>, g^{\alpha} T^{-b} \prod_{i=1}^{n} T^{-x_{i} y_{i}}, T^{d}, T^{a}\right) * g_{3}^{\vec{\rho}}$.

If $T=g_{1}^{\omega} g_{3}^{\sigma}$, from the $\mathcal{B}$ 's point of view, the $m s k$ is properly distributed because no $G_{p_{2}}$ component is in $T$.

If $T=g_{1}^{\omega} g_{2}^{\kappa} g_{3}^{\sigma}$, the $m s k$ is semi-functional and the corresponding parameters are $\vec{\imath}=<\kappa d y_{1}$, $\ldots, \kappa d y_{n}>, l_{1}=-\kappa\left(b+\sum_{i=1}^{n} x_{i} y_{i}\right), l_{2}=\kappa d$ and $l_{3}=\kappa a$. 
Because $y_{1}, \ldots, y_{n}, x_{1}, \ldots, x_{n}, d, a, b$ are merely calculated by modulo $p_{1}$ in $m p k$, from the $\mathcal{A}_{1}$ 's point of view, they are random by modulo $p_{2}$. Thus, the SF $m s k$ is distributed uniformly at random.

Oracle Query: $\mathcal{B}$ uses $g_{2} g_{3}$ to re-randomize the $G_{p_{2} p_{3}}$. Similarly, $\mathcal{B}$ uses $g_{3}$ to re-randomize the $G_{p_{3}}$. Thus, $\mathcal{B}$ is able to create the corresponding semi-functional keys by using the semi-functional master secret key.

Challenge: The phase is the same as that of Lemma 4.

Because the $m s k$ is NSF for the challenge ciphertext, we must make further efforts to prove that NSF and SF $m s k$ are indistinguishable from the $\mathcal{A}_{1}$ 's point of view in the case of the leakage of msk.

Lemma 8. The advantage that $\mathcal{A} 1$ wins when the msk is adjusted from normal SF to NSF has a negligible difference in the case of $\lambda_{m s k}$ leakage of the master secret key where $\lambda_{m s k}=(n-2 c-1) \lambda$ and $c$ is a fixed positive constant.

Proof. The proof is very similar to that of Lemma 5 except for the following differences. Note that in Lemma 5 the $G_{p_{2}}$ part of the certificate/decryption key is $(\vec{\Gamma}, 0)+\left(0, \ldots, 0, r_{1}, r_{2}\right)$. For the $m s k$ in Lemma 8 the $G_{p_{2}}$ part is $(\vec{\Gamma}, 0,0)+\left(0, \ldots, 0, r_{1}, r_{2}, 0\right)+(0, \ldots, 0, \theta)$ where $r_{1}, r_{2}, \in Z_{p_{2}}$.

When $\mathcal{A}_{1}$ gives the challenge message for the challenge identity $I D^{*}$ to $\mathcal{B}, \mathcal{B}$ chooses $t_{2} \in Z_{p_{2}}$ such that $\delta_{n+1}\left(r_{1}-I D^{*} \theta\right)+t_{2} r_{2} \equiv 0 \bmod p_{2}$. By using the output of the adversary $\mathcal{A}_{1}$ we succeed in distinguishing $\left(\vec{\delta}, f\left(\vec{\tau}^{\prime}\right)\right)$ and $(\vec{\delta}, f(\vec{\tau}))$ with non-negligible advantage. Thus, there is a contradiction.

By Lemma 7 and Lemma 8 , we have $\left|A d v_{\mathcal{A}_{1}}^{\mathcal{T}_{1} \text { Game }} Q_{-}\left(\lambda_{d k}, \lambda_{m s k}\right)-A d v_{\mathcal{A}_{1}}^{\mathcal{T}_{1} \text { Game }}{ }_{-M s k}\left(\lambda_{d k}, \lambda_{m s k}\right)\right| \leq \varepsilon$.

Lemma 9. Under the assumption 3 the advantage that PPT adversary succeeds in distinguishing $\mathcal{T}_{1}$ Game_Msk and $\mathcal{T}_{1}$ Game_Final is negligible. That is

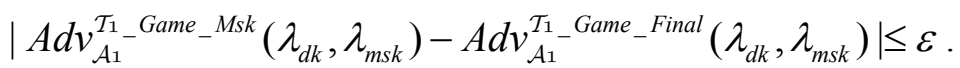

Proof. If there is a PPT adversary $\mathcal{A}_{1}$ who may succeed in distinguishing $\mathcal{T}_{1}$ Game_Msk and $\mathcal{T}_{1}$ Game_Final with non-negligible advantage, we are able to construct a simulator $\mathcal{B}$ to break the assumption 3. $\mathcal{B}$ is given an instance $\left(N, G, G_{T}, e, g_{1}, g_{2}, g_{3}, g_{1}^{\alpha} g_{2}^{v}, g_{1}^{s} g_{2}^{u}\right) . \mathcal{B}$ is given a challenge item $T$ which is $g_{1}^{\alpha s}$ or a random value in $G . \mathcal{B}$ interacts with $\mathcal{A}_{1}$ as follows.

Setup: $\mathcal{B}$ selects randomly $\alpha, x_{1}, \ldots, x_{n}, a, b, d \in Z_{N}$ and sets the master public key mpk $=\left(e\left(g_{1}^{\alpha} g_{2}^{v}, v_{1}\right), g_{1}^{x_{i}}, u_{1}=g_{1}^{a}, h_{1}=g_{1}^{b}, v_{1}=g_{1}^{d}\right) . \mathcal{B}$ selects randomly $y_{1}, \ldots, y_{n}, r \in Z_{N}$ and two vectors $\vec{\rho}, \vec{\gamma} \in Z_{N}^{n+3}$ and calculates the SF $m s k=\left(<v_{1}^{y_{1}}, \ldots, v_{1}^{y_{n}}>, h_{1}^{-r} \prod_{i=1}^{n} g_{1}^{-x_{i} y_{i}}, v_{1}^{r}, u_{1}^{r}\right) * g_{2}^{\vec{\gamma}} * g_{3}^{\vec{\rho}}$.

Oracle Query: By using the SF master secret key, $\mathcal{B}$ simulates all oracles.

Challenge: $\mathcal{A} 1$ gives an identity $I D^{*}$ and two messages $M_{0}$ and $M_{1}$ to $\mathcal{B}$. $\mathcal{B}$ selects a random bit $\xi \in\{0,1\} . \mathcal{B}$ scans the list $\mathcal{L}_{1}$ to find $p k_{I D^{*}}=(Y, \pi)$ about $I D^{*}$ for the largest handle where $Y=e\left(g_{1}, v_{1}\right)^{\alpha \beta^{*}}$. If the $\mathcal{A} 1$ doesn't replace the public key $\mathcal{B}$ knows $\beta^{*}$. Otherwise, $\mathcal{B}$ can extract $\beta^{*}$ from $\pi$ by NIZK proof. By using $g_{1}^{s} g_{2}^{u}$ and $T$ given from the assumption $\mathcal{B}$ outputs the SF ciphertext $C^{*}=\left(C_{0}^{*}, \vec{C}^{*}, C_{1}^{*}, C_{2}^{*}\right)=\left(M_{\xi}^{*} \cdot e\left(T, v_{1}^{\beta^{*}}\right),\left(g_{1}^{s} g_{2}^{\mu}\right)^{x_{i}},\left(g_{1}^{s} g_{2}^{\mu}\right)^{d}\right.$, $\left.\left(g_{1}^{s} g_{2}^{\mu}\right)^{a I D^{*}+b}\right)$.

From the $\mathcal{A}_{1}$ 's point of view, $x_{1}, \ldots, x_{n}, d, a, b$ are random under modulo $p_{2}$. So, the given SF ciphertext is distributed uniformly at random. 
If $T=g_{1}^{\alpha s}$, the ciphertext is distributed uniformly at random. In this case, $\mathcal{B}$ correctly simulates the $\mathcal{T}_{1} \_$Game_Msk. If $T$ is a random element of $G$, the item $C_{0}^{*}$ is random. Thus the ciphertext is $\mathrm{SF}$ for a random message. In this case, $\mathcal{B}$ correctly simulates the $\mathcal{T}_{1}$ _Game _Final . $\mathcal{B}$ can break assumption 3 by using the output of $\mathcal{A}_{1}$. There is a contraction. Thus, we have $\left|A d v_{\mathcal{A}_{1}}^{\mathcal{T}_{1} \text { Game }}{ }_{-}^{M s k}\left(\lambda_{d k}, \lambda_{m s k}\right)-A d v_{\mathcal{A}_{1}}^{\mathcal{T}_{1} \text { Game_Final }}\left(\lambda_{d k}, \lambda_{m s k}\right)\right| \leq \varepsilon$.

All in all, under the above nine lemmas we have that $\mathcal{T}_{1}$ Game $R$ and $\mathcal{T}_{1}$ Game Final are indistinguishable from the adversary's point of view. What's more, $A d v_{\mathcal{A}_{1}}^{T_{1} \text { Game }}{ }_{- \text {Final }}\left(\lambda_{d k}, \lambda_{m s k}\right) \leq \varepsilon$ is proved in Theorem 6.8 of the full version of [26]. To sum up, $A d v_{\mathcal{A}_{1}}^{\mathcal{T}_{1} \text { Game }{ }_{-} R}\left(\lambda_{d k}, \lambda_{m s k}\right) \leq \varepsilon$. In addition, Lemma 1 proves the leakage bound. Thus, we finish the proof of Theorem 1.

\subsubsection{Security Proof against Type II Adversary}

Theorem 2. Under assumption 1, assumption 2 and assumption 3, the LR-CBE scheme is secure in the case of $\lambda_{d k}$ leakage of the decryption key against $\mathcal{A}_{2}$, where the amount of the leakage is at most $\lambda_{d k}=(n-2 c-1) \lambda$.

Proof. The proof of the Theorem 2 is similar to that of Theorem 1 . The concrete proofs are given in Appendix A.

\section{Leakage Bound}

Our scheme is resilient to the $\lambda_{m s k}$ leakage of the master secret key and the $\lambda_{d k}$ leakage of the decryption key. The $\lambda_{m s k}$ and $\lambda_{d k}$ have the same maximum value $(n-2 c-1) \lambda$, where $n \geq 2$ is an integer and $c$ is a fixed positive constant. The leakage is subject to the size of the subgroup $G_{p_{2}}$. The value of $n$ can be varied.

In our system $N=p_{1} p_{2} p_{3}$ and $p_{1}, p_{2}, p_{3}$ are $\lambda$-bit primes. The size of the master secret key is $(n+3)(\lambda+\lambda+\lambda)=3(n+3) \lambda$. Similarly, the size of the decryption key is $3(n+2) \lambda$. The leakage rate of master secret key is $\frac{(n-2 c-1) \lambda}{3(n+3) \lambda}=\frac{(n-2 c-1)}{3(n+3)}$. The leakage rate of decryption key is $\frac{(n-2 c-1) \lambda}{3(n+2) \lambda}=\frac{(n-2 c-1)}{3(n+2)}$.

The value of $n$ can be varied and thus we obtain variable key size and variable leakage amount. The bigger value of $n$ will allow us to get the higher leakage rate. Thus we can achieve the stronger security. The smaller value of $n$ will lead to the smaller master public key. The leakage rate is close to $1 / 3$ if $n$ is large enough.

Theoretically speaking, we can get a very high leakage rate when $n$ is large enough. The leakage rate of our scheme can come up to $1 / 3$ which far exceeds that of the scheme [20]. In view of engineering practice, we hope that $n$ is a small value. We can see that when $n=4$ the leakage rate of our scheme can come up to $1 / 6$ which already reaches that of the scheme [20]. What's more, we need only 6 pairings in our decryption which is acceptable in decryption calculation. In some settings, if the leakage is not serious we can even set $n=2$. Even so, the leakage rate of our scheme is $1 / 12$ which is slightly good. Thus, our scheme is also practically usable if we select the small $n$.

\section{Comparisons}

We compare our scheme with the schemes in $[1,5]$. There are two CBE schemes in [1], BasicCBE and FullCBE, neither of which has leakage resilience. The major contribution of [5] is a key encapsulation mechanism which can be used to construct CBE. The key encapsulation mechanism has no leakage resilience either. Our scheme is a practical and secure scheme with leakage resilience. Denote the hash operation by $H$ and the pairing computation by $P$. The calculations of encryption 
and decryption of the above schemes are given in the following table 3 .

Table 3. Performance comparison of our scheme and schemes in $[1,5]$

\begin{tabular}{|l|l|l|l|}
\hline Schemes & Encryption Calculation & Decryption Calculation & Leakage Resilience \\
\hline BasicCBE in $[1]$ & $3 H+2 P$ & $1 H+1 P$ & No \\
\hline FullCBE in [1] & $4 H+2 P$ & $3 H+1 P$ & No \\
\hline Scheme in [5] & $1 H+1 P$ & $1 H+1 P$ & No \\
\hline Our scheme & $1 H+1 P$ & $1 H+(n+2) P$ & Yes \\
\hline
\end{tabular}

As shown in table 3, for the encryption calculation, our scheme is as good as that of the work in [5].

What's more, the value of $n$ has an important impact on the decryption calculation of our scheme.

The value of $n$ also determines the key leakage rate. From table 3, we can see that a bigger $n$ will lead to more decryption operations in our scheme. But as shown in Section 6, a bigger $n$ will help us to achieve a higher leakage rate (and stronger security). In practice, we should make a trade off between security and computational overhead in encryption and decryption.

When $n$ varies, the following table 4 gives the corresponding changes of the decryption calculation and leakage rate.

Table 4. The changes of leakage rate and decryption calculation about the parameter $n$.

\begin{tabular}{|c|c|c|c|c|c|c|c|c|}
\hline$n$ & 2 & 3 & 4 & 5 & $\ldots \ldots$ & $n$ & $\ldots \ldots$ & $+\infty$ \\
\hline Pairings of decryption & 4 & 5 & 6 & 7 & $\ldots \ldots$ & $n+2$ & $\ldots \ldots$ & $+\infty$ \\
\hline Leakage rate & $\frac{1}{12}$ & $\frac{2}{15}$ & $\frac{1}{6}$ & $\frac{4}{21}$ & $\ldots \ldots$ & $\frac{n-1}{3(n+2)}$ & $\ldots \ldots$ & $\frac{1}{3}$ \\
\hline
\end{tabular}

We can see from the table 3 that the decryption needs $n+2$ pairings. According to table 4 , we will know the following fact easily. When $n$ is very large, both the decryption overhead and the leakage rate are very large. On the other hand, when the $n$ is smaller, the key leakage rate is smaller and accordingly the decryption overhead is smaller. Even $n=2$ is a very small valve, the key leakage rate may come to $\frac{1}{12}$ which can afford the better leakage resilience in practice. At the same time, when $n=2$ the decryption only needs 4 pairings which is acceptable in practical application. To say the least, when $n=4$ the leakage rate may amount to $\frac{1}{6}$ which affords a very good leakage resilience ( please refer to [20] ), but even so the decryption needs no more than 6 pairings which is also acceptable for engineering practice. Thus, we may select smaller value of $n$ for the practicality, such as $n=2, n=3$ or $n=4$. At the same time, the leakage resilience of our scheme is very good.

\section{Conclusion}

Formal definitions and security models for LR-CBE are given in this paper. We present a leakage-resilient certificate-based encryption scheme in which leakage about the decryption key and the master secret key is considered. The security of the scheme is reduced to the composite order bilinear groups assumption. To the best of our knowledge, this is the first LR-CBE resilient to master secret key leakage. Our scheme has good leakage resilience. The leakage rate is close to $1 / 3$ if we adjust $n$ properly. Performance analysis shows our scheme has low computation overhead in encryption phase. To improve efficiency for decryption operation, we can select $n=2$ and decryption operation only needs 4 pairings which is acceptable in practical applications. As a direction of future work, we will construct secure LR-CBE under standard complexity assumptions such as prime order bilinear groups assumption, which can make the scheme more efficient. 


\section{Acknowledgements}

We would like to thank anonymous referees for their helpful comments and suggestions to improve our paper. This research is supported by the National Natural Science Foundation of China (61272542, 61472083, 61402110, 61170298), the Fundamental Research Funds for the Central Universities (2013B07014), the Funding of Jiangsu Innovation Program for Graduate Education (KYZZ_0139) and the Natural Science Foundation of the Jiangsu Higher Education Institutions of China (14KJD520006), Fok Ying Tung Education Foundation, Ministry of Education, China (141065), Ph.D. Programs Foundation of Ministry of Education of China (20123503120001), Program for New Century Excellent Talents in Fujian University (JA14067), Distinguished Young Scholars Fund of Department of Education, Fujian Province, China (JA13062).

\section{References}

[1] Gentry C., 2003. Certificate-based encryption and the certificate revocation problem. In: Biham, E. (Ed.), EUROCRYPT. Vol. 2656 of Lecture Notes in Computer Science. Springer, pp. 272-293.

[2] Li J., Wang Z., Zhang Y., 2013. Provably secure certificate-based signature scheme without pairings. Information Sciences 233 (6), 313-320.

[3] Li J., Huang X., Hong M., Zhang Y., 2012. Certificate-based signcryption with enhanced security features. Computers and Mathematics with Applications 64 (6), 1587-1601.

[4] Li J., Huang X., Zhang Y., Xu L., 2012. An efficient short certificate-based signature scheme. Journal of Systems and Software 85 (2), 314-322.

[5] Li J., Yang H., Zhang Y., 2012. Certificate-based key encapsulation mechanism with tags. Journal of Software 23 (8), 2163-2172.

[6] Li J., Huang X., Mu Y., Susilo W, Wu Q., 2010. Constructions of certificate-based signature secure against key replacement attacks. Journal of Computer Security 18 (3), 421-449.

[7] Lu Y., Li J., 2012. Constructing certificate-based encryption secure against key replacement attacks. ICIC Express Letters, Part B: Applications 3 (1), 195-200.

[8] Lu Y., Li J., 2010. Forward-secure certificate-based encryption and its generic construction. Journal of Networks 5 (5), 527-534.

[9] Halderman J.A., Schoen S.D., Heninger N., et al. 2009. Lest we remember: cold-boot attacks on encryption keys. Communications of the ACM 52(5), 91-98.

[10] Dodis Y., Pietrzak K., 2010. Leakage-resilient pseudorandom functions and side-channel attacks on Feistel networks. In: Rabin T. (Ed.), CRYPTO. Vol. 6223 of Lecture Notes in Computer Science. Springer, pp. 21-40.

[11] Brumley D., Boneh D., 2005. Remote timing attacks are practical. Computer Networks 48 (5), 701-716.

[12] Gandolfi K., Mourtel C., Olivier F., 2001. Electromagnetic analysis: concrete results. In: Koç C.K., Naccache D., Paar C. (Eds), CHES. Vol. 2162 of Lecture Notes in Computer Science Volume. Springer, pp. 251-261.

[13] Chen C., Wang T., Tian J., 2013. Improving timing attack on rsa-crt via error detection and correction strategy. Information Sciences 232, 464-474.

[14] Micali S., Reyzin L., 2004. Physically observable cryptography. In: Naor M. (Ed.), TCC. Vol. 2951 of Lecture Notes in Computer Science. Springer, pp. 278-296.

[15] Pietrzak K., 2009. A leakage-resilient mode of operation. In: Joux A. (Ed.), EUROCRYPT. Vol. 5479 of Lecture Notes in Computer Science. Springer, pp. 462-482.

[16] Dziembowski S., Pietrzak K., 2008. Leakage-resilient cryptography. In: (Ed.), FOCS. IEEE, pp. 293-302.

[17] Faust S., Kiltz E., Pietrzak K., et al., 2010. Leakage-resilient signatures. In: Micciancio D. (Ed.), TCC. Vol. 5978 of Lecture Notes in Computer Science. Springer, pp. 343-360.

[18] Akavia A., Goldwasser S., VaikuntanathanV., 2009. Simultaneous hardcore bits and cryptography against memory attacks. In: Reingold O. (Ed.) TCC. Vol. 5444 of Lecture Notes in Computer Science. Springer, pp. 474-495.

[19] Chow S.M., Dodis Y., Rouselakis Y., Waters B, 2010. Practical leakage-resilient identity-based encryption from simple assumptions. In: CCS. ACM, pp. 152-161.

[20] Naor M., Segev G., 2012. Public-key cryptosystems resilient to key leakage. SIAM Journal on Computing 41 (4), 772-814.

[21] Katz J., Vaikuntanathan V., 2009. Signature schemes with bounded leakage resilience. In: Matsui 
M. (Ed.), ASIACRYPT. Vol. 5912 of Lecture Notes in Computer Science. Springer, pp. 703-720.

[22] Alwen J., Dodis Y., Wichs D., 2009. Leakage-resilient public-key cryptography in the bounded-retrieval model. In: Halevi S, (Ed.), CRYPTO. Vol. 5677 of Lecture Notes in Computer Science. Springer, pp. 36-54.

[23] Chen Y., Luo S., Chen Z., 2011. A new leakage-resilient ibe scheme in the relative leakage model. In: Li Y. (Ed.), Data and Applications Security and Privacy XXV. Vol. 6818 of Lecture Notes in Computer Science. Springer, pp. 263-270.

[24] Luo X., Qian P., Zhu Y., Wang S., 2010. Leakage-resilient identity-based encryption scheme. In: (Ed.), IEEE, pp. 324-329.

[25] Alwen J., Dodis Y., Naor M., et al., 2010. Public-key encryption in the bounded-retrieval model. In: Gilbert H. (Ed.), EUROCRYPT. Vol. 6110 of Lecture Notes in Computer Science. Springer, pp. $113-134$.

[26] Lewko B., Rouselakis Y., Waters B., 2011. Achieving leakage resilience through dual system encryption. In: Ishai Y. (Ed.), TCC. Vol. 6597 of Lecture Notes in Computer Science. Springer, pp. $70-88$.

[27] Xiong H., Yuen T.H., Zhang C., Yiu S.M., He Y.J., 2013. Leakage-resilient certificateless public key encryption. In: AsiaPKC 2013, ACM, pp. 13-22.

[28] Wu W., Mu Y., Susilo W., Huang X., Xu L., 2012. A provably secure construction of certificate-based encryption from certificateless encryption. Comput. J. 55 (10), 1157-1168.

[29] Waters B., 2009. Dual system encryption: Realizing fully secure ibe and hibe under simple assumptions. In: Halevi S. (Ed.), CRYPTO. Vol. 5677 of Lecture Notes in Computer Science. Springer, pp. 619-636.

[30] Lewko A., Waters B., 2010. New techniques for dual system encryption and fully secure hibe with short ciphertexts. In: Micciancio D. (Ed.), TCC. Vol. 5978 of Lecture Notes in Computer Science. Springer, pp. 455-479.

[31] Groth J., 2010. Short non-interactive zero-knowledge proofs. In: Abe M. (Ed.), ASIACRYPT. Vol. 6477 of Lecture Notes in Computer Science Volume. Springer, pp. 341-358.

[32] Boneh D., Goh E., Nissim K., 2005. Evaluating 2-dnf formulas on ciphertexts. In: Kilian J. (Ed.), TCC. Vol. 3378 of Lecture Notes in Computer Science. Springer, pp. 325-341.

[33] Brakerski Z., Kalai Y.T., Katz J., Vaikuntanathan V., 2010. Overcoming the hole in the bucket: Public-key cryptography resilient to continual memory leakage. In: FOCS. IEEE, pp. 501-510.

Appendix A. Proof of Theorem 2

In order to prove the security with leakage resilience against Type II adversary, we give a series of games here. These games are modifications of the game $\mathcal{T}_{2}$ Game $R$. Thus, if we prove that these games are indistinguishable we complete the proof of Theorem 2. We use $Q$ to denote the maximum number of $\mathcal{O}$-Create queries in the games.

These additional games are as follows.

The hybrid games are as follows.

$\mathcal{T}_{2}$ _Game_0 : It is nearly the same as $\mathcal{T}_{2}$ _Game_ $R$ besides the challenge ciphertext. In $\mathcal{T}_{2}$ Game_O the challenge ciphertext is semi-functional.

$\mathcal{T}_{2} \_$Game_ $k(k=1$ to $Q)$ : In the game, for the adversary's queries, the challenger answers in two ways.

Towards the first $k$ queries, the challenger answers with the SF certificate and SF decryption key. The challenger adds the corresponding item in list $\mathcal{L}_{2}$ and gives it to the adversary.

Towards the remaining queries, the challenger answers with normal certificate and normal decryption key.

$\mathcal{T}_{2}$ Game_Final : It is nearly the same as $\mathcal{T}_{2}$ Game $Q$ except that in the game the challenger selects randomly a message $M_{\zeta}$ and encrypts it. In $\mathcal{T}_{2} \_$Game_ $Q$, the challenge encrypts a challenge message $M_{\xi}$. From the adversary's point of view, in $\mathcal{T}_{2}$ Game_Final the bit $\xi^{\prime}$ of his guess is independent of the bit $\xi$. 
In the following table 5, we explain the types of ciphertexts and decryption keys which are created in different games. Let SF denote semi-functional key or ciphertext. Let $\mathrm{N}$ denote normal key or ciphertext. We use $T_{C}$ and $T_{D}$ to denote the types of ciphertext and decryption key, respectively. In each game, the maximum number of create query $\mathcal{O}$-Create is $Q$. Thus, we use $(\underbrace{\left(\mathrm{T}_{\mathrm{C}}, \mathrm{T}_{\mathrm{D}}\right), \ldots,\left(\mathrm{T}_{\mathrm{C}}, \mathrm{T}_{\mathrm{D}}\right)}_{Q})$ to denote the corresponding types of $Q$ create queries in a game. For every game above, the types of the ciphertexts are the same in every create query. Thus, $\left(\left(\mathrm{T}_{\mathrm{C}}, \mathrm{T}_{\mathrm{D}}\right), \ldots,\left(\mathrm{T}_{\mathrm{C}}, \mathrm{T}_{\mathrm{D}}\right)\right)$ may be shortened as $\left(\mathrm{T}_{\mathrm{C}},(\underbrace{\mathrm{T}_{\mathrm{D}}, \ldots \mathrm{T}_{\mathrm{D}}}_{Q})\right)$ to denote the according types of $Q$ create queries in a game.

Table 5. The types of ciphertexts and decryption keys in different games.

\begin{tabular}{|c|c|}
\hline Games & The type of ciphertexts and decryption keys: $\left(\mathrm{T}_{\mathrm{C}},\left(\mathrm{T}_{\mathrm{D}}, \ldots, \mathrm{T}_{\mathrm{D}}\right)\right)$ \\
\hline $\mathcal{T}_{2} \_$Game_ $R$ & $(\mathrm{~N},(\mathrm{~N}, \ldots, \mathrm{N}))$ \\
\hline $\mathcal{T}_{2} \_$Game_o & $(\mathrm{SF},(\mathrm{N}, \ldots \ldots, \mathrm{N}))$ \\
\hline $\mathcal{T}_{2} \_$Game_k & $(\mathrm{SF},(\mathrm{SF}, \ldots, \underset{\mathrm{k}}{\mathrm{S}}, \mathrm{N}, \ldots, \mathrm{N}))$ \\
$k \in(1, \ldots, Q-1)$ & $(\mathrm{SF},(\mathrm{SF}, . ., \mathrm{SF}))$ \\
\hline $\mathcal{T}_{2} \_$Game_Q & $(\mathrm{SF},(\mathrm{SF}, \ldots, \mathrm{SF}))$ \\
\hline $\mathcal{T}_{2}$ Game_Final & \\
\hline
\end{tabular}

Theorem 2. Under assumption 1, assumption 2 and assumption 3, the LR-CBE scheme is secure in the case of $\lambda_{d k}$ leakage of the decryption key against $\mathcal{A}_{2}$, where the amount of the leakage is at most $\lambda_{d k}=(n-2 c-1) \lambda$.

Proof. The general idea of proof is as follows. We will use a series of games $\mathcal{T}_{2}$ Game $\_$, $\mathcal{T}_{2} \_$Game_k $(k \in(0,1, \ldots, Q))$ and $\mathcal{T}_{2} \_$Game_Final and Lemma 10 to Lemma 16 to finish our security proof. We will use the following Lemma 10 to Lemma 16 to prove the security concretely. On one hand, we prove that these games are indistinguishable by Lemma 11 to Lemma 16. On the other hand, the advantage that the adversary wins in $\mathcal{T}_{2}$ _Game _ Final is negligible. We get the leakage bound by Lemma 10. Thus, we can obtain the security of the scheme.

A bit more specifically, we use the following table 6 to show the difference of advantages that the adversary wins in the successive two games. Thus, we are easy to obtain the security. Here, we only use the results of Lemma 10 to Lemma 16. The specific proofs of Lemma 10 to Lemma 16 are given in the following. Let $A d v_{\mathcal{A}_{2}}^{\mathcal{T}_{2} \text { Game }{ }_{-}{ }^{k}}\left(\lambda_{d k}\right)$ denote the advantage that the adversary $\mathcal{A}_{2}$ wins in game $\mathcal{T}_{2}$ Game_ $k(k \in(1, \ldots, Q))$. Let $A d v_{\mathcal{A}_{2}^{-}}^{\mathcal{T}_{2} \text { Game }}{ }_{-}$Final $\left(\lambda_{d k}\right)$ denote the advantage that the adversary $\mathcal{A}_{2}$ wins in game $\mathcal{T}_{2}$ _Game_Final.

Table 6 . The difference of advantages that the adversary wins in the successive two games.

\begin{tabular}{|c|c|c|}
\hline Two successive games & Difference of advantages & $\begin{array}{l}\text { Related } \\
\text { lemmas }\end{array}$ \\
\hline $\begin{array}{l}\mathcal{T}_{2} \text { Game_R } \\
\mathcal{T}_{2} \text { _Game_0 }\end{array}$ & $\left|A d v_{\mathcal{A}_{2}^{-}}^{\mathcal{T}_{2} \text { Game }{ }_{-}{ }^{R}}\left(\lambda_{d k}\right)-A d v_{\mathcal{A}_{2}^{-}}^{\mathcal{T}_{2^{-}} \text {Game }_{-}{ }^{0}}\left(\lambda_{d k}\right)\right| \leq \varepsilon$ & Lemma 11 \\
\hline $\begin{array}{l}\mathcal{T}_{2} \_ \text {Game_k } \\
\mathcal{T}_{2} \text { _Game_k+1 } \\
k \in(0,1, \ldots, Q-1)\end{array}$ & $\left|A d v_{\mathcal{A}_{2}^{-}}^{\mathcal{T}_{2} \text { Game }{ }_{-}{ }^{k}}\left(\lambda_{d k}\right)-A d v_{\mathcal{A}_{2}}^{\mathcal{T}_{2} \text { Game }_{-}{ }^{k+1}}\left(\lambda_{d k}\right)\right| \leq \varepsilon$ & $\begin{array}{l}\text { Lemma } 12 \\
\text { Lemma } 13 \\
\text { Lemma } 14 \\
\text { Lemma } 15\end{array}$ \\
\hline $\begin{array}{l}\mathcal{T}_{2} \text { _Game_Q } Q \text { and } \\
\mathcal{T}_{2} \text { _Game_Final }\end{array}$ & 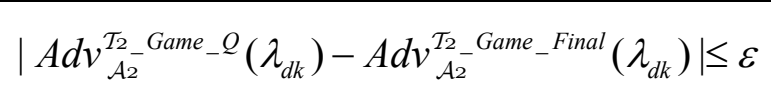 & Lemma 16 \\
\hline
\end{tabular}

From table 6 , the security of our scheme is obtained directly. We have: 


$$
\begin{aligned}
& \left|A d v_{\mathcal{A}_{2}^{-}}^{\mathcal{T}_{2} \text { Game }}{ }^{R}\left(\lambda_{d k}\right)-A d v_{\mathcal{A}_{2}^{-}}^{\mathcal{T}_{2} \text { Game_Final }}\left(\lambda_{d k}\right)\right| \\
& \mid A d v_{\mathcal{A}_{2}}^{\mathcal{T}_{2} \text { Game }{ }_{-} R}\left(\lambda_{d k}\right)-A d v_{\mathcal{A}_{2}}^{\mathcal{T}_{2} \text { Game }}{ }^{0}\left(\lambda_{d k}\right)+A d v_{\mathcal{A}_{2}}^{\mathcal{T}_{2} \text { Game }{ }_{-}{ }^{0}}\left(\lambda_{d k}\right)-\ldots \\
& =-A d v_{\mathcal{A}_{2}}^{\mathcal{T}_{2_{-}} \text {Game }{ }_{-} k}\left(\lambda_{d k}\right)+A d v_{\mathcal{A}_{-}}^{\mathcal{T}_{2} \text { Game }{ }_{-} k}\left(\lambda_{d k}\right)-\ldots A d v_{\mathcal{A}_{2}}^{\mathcal{T}_{2} \text { Game }{ }_{-} k+1}\left(\lambda_{d k}\right)+\ldots
\end{aligned}
$$

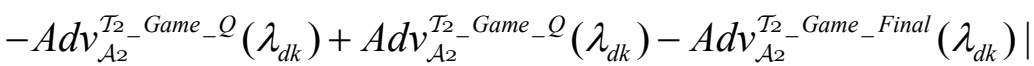

$$
\begin{aligned}
& \left|A d v_{\mathcal{A}_{1}}^{\mathcal{T}_{2_{-}} \text {Game }{ }_{-}{ }^{R}}\left(\lambda_{d k}, \lambda_{m s k}\right)-A d v_{\mathcal{A}_{1}}^{\mathcal{T}_{2_{-}} \text {Game }{ }_{-}{ }^{0}}\left(\lambda_{d k}, \lambda_{m s k}\right)\right| \\
& \leq+\left|A d v_{\mathcal{A}_{2}}^{\mathcal{T}_{2_{-}} \text {Game }{ }_{-} 0}\left(\lambda_{d k}\right)-A d v_{\mathcal{A}_{2}}^{\mathcal{T}_{2} \text { Game }{ }_{-} 1}\left(\lambda_{d k}\right)\right|+\ldots \\
& +\left|A d v_{\mathcal{A}_{2}}^{\mathcal{T}_{2} \text { Game }{ }_{-}{ }^{2}}\left(\lambda_{d k}\right)-A d v_{\mathcal{A}_{2}}^{\mathcal{T}_{2} \text { Game }{ }_{-} k+1}\left(\lambda_{d k}\right)\right|+\ldots \\
& +\mid A d v_{\mathcal{A}_{2}}^{\mathcal{T}_{2} \text { Game } Q_{-}}\left(\lambda_{d k}\right)-A d v_{\mathcal{A}_{2}}^{\mathcal{T}_{2} \text { Game }} \text { Final }\left(\lambda_{d k}\right) \mid \\
& \leq \varepsilon+(\mathrm{Q}+1) \varepsilon=(\mathrm{Q}+2) \varepsilon \text {. }
\end{aligned}
$$

So, $\quad \mid A d v_{\mathcal{A}_{2}}^{\mathcal{T}_{2} \text { Game }{ }_{-}{ }^{R}}\left(\lambda_{d k}\right)-A d v_{\mathcal{A}_{2}}^{\mathcal{T}_{2} \text { Game }}$ Final $\left(\lambda_{d k}\right) \mid \leq(Q+2) \varepsilon \quad$. What's more, $A d v_{\mathcal{A}_{2}}^{\mathcal{T}_{2} \text { Game }}$ Final $\left(\lambda_{d k}\right) \leq \varepsilon$ is proved similarly to that of Theorem 6.8 in the full version of [26]. To sum up, $A d v_{\mathcal{A}_{2}}^{\mathcal{T}_{2} \text { Game }_{-}{ }^{R}}\left(\lambda_{d k}\right) \leq \varepsilon$. In addition, Lemma 10 proves the leakage bound. Thus, we finish the proof of Theorem 2.

The specific proofs of Lemma 10 to Lemma 16 are given as follows.

Lemma 10. The leakage size is at most $\lambda_{d k}=(n-2 c-1) \lambda$.

Proof. The proof of the lemma is identical to the proof of Lemma 1.

Lemma 11. Under assumption 1, the advantage that PPT adversary $\mathcal{A}_{2}$ succeeds in distinguishing $\mathcal{T}_{2}$ Game $R$ and $R$ T $\mathcal{T}_{-}$Game_ 0 is negligible. That is $\left|A d v_{\mathcal{A}_{2}^{-}}^{\mathcal{T}_{2} \text { Game }{ }_{-} R}\left(\lambda_{d k}\right)-A d v_{\mathcal{A}_{2}^{-}}^{\mathcal{T}_{2} \text { Game }}{ }^{0}\left(\lambda_{d k}\right)\right| \leq \varepsilon$.

Proof. If there is a PPT adversary $\mathcal{A}_{2}$ who distinguishes $\mathcal{T}_{2}$ Game $R$ and $\mathcal{T}_{2}$ Game_0 with non-negligible advantage, we can construct a simulator $\mathcal{B}$ to break assumption 1 .

Firstly, $\mathcal{B}$ is given an instance $\left(N, G, G_{T}, e, g_{1}, g_{3}\right)$ and a challenge item $T$ where $T=g_{1}^{z}$ or $T=g_{1}^{z} g_{2}^{v}$ for $z, v \in Z_{N}$. $\mathcal{B}$ interacts with $\mathcal{A}_{2}$ as follows.

Setup Phase: $\mathcal{B}$ firstly creates composite order bilinear groups $\left(N=p_{1} p_{2} p_{3}, G, G_{T}, e\right)$. Then, $\mathcal{B}$ selects $a, b, d \in Z_{N}$ uniformly at random and sets $u_{1}=g_{1}^{a}, h_{1}=g_{1}^{b}$ and $v_{1}=g_{1}^{d}$. Given $g_{1} \in G_{p_{1}}$ and $g_{3} \in G_{p_{3}}, \mathcal{B}$ runs $G e n$ of $\prod$ to generate the common reference string crs and selects random $\left(\alpha, x_{1}, x_{2}, \ldots, x_{n}, r, y_{1}, y_{2}, \ldots, y_{n}\right) \in Z_{N}^{2 n+2}$ and a vector $\vec{\rho}=<\rho_{1}, \rho_{2}, \ldots, \rho_{n+3}>$ $\in Z_{N}^{n+3}$ where $n \geq 2$ is an integer. It outputs the master public key $m p k=\left(N, G, G_{T}, e, e\left(g_{1}, v_{1}\right)^{\alpha}\right.$, $\left.g_{1}, g_{1}^{x_{1}}, \ldots, g_{1}^{x_{n}}, u_{1}, h_{1}, v_{1}, g_{3}, c r s\right)$ and the master secret key $m s k=\left(\vec{K}, K_{1}, K_{2}, K_{3}\right)$ $=\left(<v_{1}^{y_{1}}, \ldots, v_{1}^{y_{n}}>, g_{1}^{\alpha} h_{1}^{-r} \prod_{i=1}^{n} g_{1}^{-x_{i} y_{i}}, v_{1}^{r}, u_{1}^{r}\right) * g_{3}^{\vec{\rho}}$.

Oracle Query: $\mathcal{B}$ knows the decryption key because he has the $m s k$. Thus, $\mathcal{B}$ can answer the adversary's queries. In addition, $\mathcal{B}$ stores the private key of each user.

Challenge: $\mathcal{A}_{2}$ gives identity $I D^{*}$ and two message $M_{0}$ and $M_{1}$ to $\mathcal{B} . \mathcal{B}$ scans list $\mathcal{L}_{2}$ to find public key $p k_{I D^{*}}=(Y, \pi)$ of $I D^{*}$ where $Y=e\left(g_{1}, v_{1}\right)^{\alpha \beta}$. Because the $\mathcal{A}_{2}$ doesn't replace the public key $\mathcal{B}$ knows $\beta . \mathcal{B}$ selects $\xi \in\{0,1\}$ at random and outputs $C^{*}=\left(C_{0}^{*}, \vec{C}^{*}, C_{1}^{*}, C_{2}^{*}\right)$ $=\left(M_{\xi} \cdot e\left(v_{1}^{\beta}, T\right),<T^{x_{1}}, \ldots, T^{x_{n}}>, T^{d}, T^{a I D^{*}+b}\right)$.

When $T=g_{1}^{z}$ (that is, no $G_{p_{2}}$ part is contained in it), the given ciphertext is normal. Thus, $\mathcal{B}$ 
simulates the $\mathcal{T}_{2}$ Game $R$ correctly.

When $T=g_{1}^{z} g_{2}^{v}$, we let $\vec{\delta}=<\mathrm{x}_{1}, \ldots, \mathrm{x}_{n}>, \delta_{1}=d, \delta_{2}=a I D^{*}+b$. We see that $\vec{\delta}, \delta_{1}$ and $\delta_{2}$ are distributed uniformly at random from the adversary's view. The reason is that the $x_{1}, \ldots, x_{n}, d, a$ and $b$ are merely calculated under modulo $p_{1}$ in $m p k$. So, from the adversary's point of view, $x_{1}, \ldots, x_{n}, d, a$ and $b$ are random under modulo $p_{2}$. Thus, $\mathcal{B}$ simulates the $\mathcal{T}_{2}$ Game_0 correctly.

Therefore, if $\mathcal{A}_{2}$ can distinguish $\mathcal{T}_{2}$ Game $R$ and $\mathcal{T}_{2}{ }_{-}$Game_ 0 with non-negligible advantage, $\mathcal{B}$ breaks assumption 1 by $\mathcal{A}_{2}$ with non-negligible advantage. We have $\left|A d v_{\mathcal{A}_{2}^{-}}^{\mathcal{T}_{2} \text { Game }_{-}{ }^{R}}\left(\lambda_{d k}\right)-A d v_{\mathcal{A}_{2}^{-}}^{\mathcal{T}_{2} \text { Game }{ }_{-}{ }^{0}}\left(\lambda_{d k}\right)\right| \leq \varepsilon$.

Lemma 12. If $\lambda_{d k}=(n-2 c-1) \lambda$ and assumption 2 holds, the advantage that PPT adversary $\mathcal{A}_{2}$ distinguishes $\mathcal{T}_{2}$ Game_k and $\mathcal{T}_{2}{ }_{-}$Game_k+1 is negligible. That is $\left|A d v_{\mathcal{A}_{2}^{-}}^{\mathcal{T}_{2} \text { Game }{ }_{-}{ }^{k}}\left(\lambda_{d k}\right)-A d v_{\mathcal{A}_{2}^{-}}^{\mathcal{T}_{2} \text { Game }_{-}{ }^{k+1}}\left(\lambda_{d k}\right)\right| \leq \varepsilon$.

Proof. In order to prove the Lemma 12 , another game which is called $\mathcal{T}_{2}$ AltGame $k$ is defined. Compared with $\mathcal{T}_{2}$ Game $k_{\text {, }}$, in $\mathcal{T}_{2}$ AltGame $k$, for the $k^{\text {th }}$ query $\mathcal{B}$ answers with normal certificate. The decryption key is semi-functional. Therefore, the Lemma 12 is proved by the following three lemmas.

Lemma 13. If $\lambda_{d k}=(n-2 c-1) \lambda$ and assumption 2 holds, the advantage that PPT adversary $\mathcal{A}_{2}$ succeeds in distinguishing $\mathcal{T}_{2}$ Game_k and $\mathcal{T}_{2}$ AltGame $k$ is negligible. That is $\left|A d v_{\mathcal{A}_{2}^{-}}^{\mathcal{T}_{2} \text { Game }{ }_{-}{ }^{k}}\left(\lambda_{d k}\right)-A d v_{\mathcal{A}_{2}}^{\mathcal{T}_{2} \text { AltGame }{ }_{-}{ }^{k}}\left(\lambda_{d k}\right)\right| \leq \varepsilon$.

Proof. If there is a PPT adversary $\mathcal{A}_{2}$ who can distinguish the $\mathcal{T}_{2}$ Game $k$ and $\mathcal{T}_{2}$ A AltGame_ $k$ with non-negligible advantage, we can construct a simulator $\mathcal{B}$ who breaks assumption 2. Firstly, $\mathcal{B}$ is given an instance $\left(N, G, G_{T}, e, g_{1}, g_{3}, g_{1}^{z} g_{2}^{v}, g_{2}^{u} g_{3}^{\rho}\right)$. $\mathcal{B}$ is given a challenge item $T$ which is $g_{1}^{\omega} g_{3}^{\sigma}$ or $g_{1}^{\omega} g_{2}^{\kappa} g_{3}^{\sigma}$. $\mathcal{B}$ interacts with $\mathcal{A}_{2}$ as follows.

Setup Phase: $\mathcal{B}$ firstly creates composite order bilinear groups $\left(N=p_{1} p_{2} p_{3}, G, G_{T}, e\right) . \mathcal{B}$ selects $a, b, d \in Z_{N}$ uniformly at random and sets $u_{1}=g_{1}^{a}, h_{1}=g_{1}^{b}$ and $v_{1}=g_{1}^{d}$. Given $g_{1} \in G_{p_{1}}$ and $g_{3} \in G_{p_{3}}, \mathcal{B}$ runs $G e n$ of $\Pi$ to generate the common reference string crs and selects $\operatorname{random}\left(\alpha, x_{1}, x_{2}, \ldots, x_{n}, r, y_{1}, y_{2}, \ldots, y_{n}\right) \in Z_{N}^{2 n+2}$ and a vector $\vec{\rho}=<\rho_{1}, \rho_{2}, \ldots, \rho_{n+3}>\in Z_{N}^{n+3}$ where $n \geq 2$ is an integer.

It outputs the master public key $m p k=\left(N, G, G_{T}, e, e\left(g_{1}, v_{1}\right)^{\alpha}, g_{1}, g_{1}^{x_{1}}, \ldots, g_{1}^{x_{n}}, u_{1}, h_{1}, v_{1}, g_{3}, c r s\right)$ and the master secret key $m s k=\left(\vec{K}, K_{1}, K_{2}, K_{3}\right)=\left(\left\langle v_{1}^{y_{1}}, \ldots, v_{1}^{y_{n}}\right\rangle, g_{1}^{\alpha} h_{1}^{-r} \prod_{i=1}^{n} g_{1}^{-x_{i} y_{i}}, v_{1}^{r}, u_{1}^{r}\right) * g_{3}^{\vec{\rho}}$.

Oracle Query: $\mathcal{B}$ may reply every query because he knows $\alpha, x_{i}, y_{i}, r$. Specifically, $\mathcal{B}$ answers the $j^{\text {th }}$ query for $I D_{j}$ as follows.

For a create query, $\mathcal{B}$ generates normal certificate $\operatorname{Cert}_{I D_{j}}$ by using $m s k$. The $\operatorname{Cert}_{I D_{j}}$ can leak information. $\mathcal{B}$ runs SetPrivateKey to get $\beta$ and runs SetPublicKey to get $p k_{I D_{j}}$. $\mathcal{B}$ picks randomly $\left(\omega_{1}, \omega_{2}, \ldots, \omega_{n}, t\right) \in Z_{N}^{n+1}$ and $\vec{\rho} \in Z_{N}^{n+2}$.

(a) If $j \leq k, \mathcal{B}$ selects randomly a vector $\vec{\gamma} \in Z_{N}^{n+2}$ and calculates the decryption key $d k_{I D_{j}}$ $=\left(<v_{1}^{\omega_{1}}, \ldots, v_{1}^{\omega_{n}}>, g^{\alpha \beta}\left(u_{1}^{I D_{j}} h_{1}\right)^{-t} \prod_{i=1}^{n} g_{1}^{-x_{i} \omega_{i}}, v_{1}^{t}\right) *\left(g_{2}^{\mu} g_{3}^{\rho}\right)^{\vec{\gamma}} * g_{3}^{\vec{\rho}}$. 
(b) If $j>k+1, \mathcal{B}$ calculates the decryption key $d k_{I D_{j}}=\left(\left(v_{1}^{\omega_{1}}, \ldots, v_{1}^{\omega_{n}}\right), g^{\alpha \beta}\left(u_{1}^{I D_{j}} h_{1}\right)^{-t} \prod_{i=1}^{n} g_{1}^{-x_{i} \omega_{i}}\right.$, $\left.v_{1}^{t}\right) * g_{3}^{\vec{\rho}}$.

(c) If $j=k+1, \mathcal{B}$ calculates the decryption key $d k_{I D_{k+1}}=\left(<T^{d w_{1}}, \ldots, T^{d w_{n}}\right\rangle, T^{-\left(a I D_{k+1}+b\right)} *$ $\left.\prod_{i=1}^{n} T^{-x_{i} \omega_{i}} * g^{\alpha \beta}, T^{d}\right) * g_{3}^{\vec{\rho}}$ by using the challenge item $T$.

If $T=g_{1}^{\omega} g_{3}^{\sigma}$, from the $\mathcal{B}$ 's point of view, the decryption key is normal because no $G_{p_{2}}$ part is in $T$.

If $T=g_{1}^{\omega} g_{2}^{\kappa} g_{3}^{\sigma}$, the decryption key is semi-functional and the corresponding parameters are $\vec{\theta}=<\kappa d w_{1}, \ldots, \kappa d w_{n}>, \theta_{1}=-\kappa\left(a I D_{k+1}+b+\sum_{i=1}^{n} x_{i} w_{i}\right)$ and $\theta_{2}=\kappa d$.

Because $w_{1}, \ldots, w_{n}, x_{1}, \ldots, x_{n}, d, a, b$ are merely calculated by modulo $p_{1}$ in $m p k$, from the $\mathcal{A}_{2}$ 's point of view, they are random by modulo $p_{2}$. Thus, the SF decryption key is properly distributed.

$\mathcal{B}$ adds the item $\left(H, I D_{j}, I D_{j}^{\prime}, p k_{I D_{j}}, s k_{I D_{j}}, \operatorname{Cert}_{I D_{j}}, d k_{I D_{j}}, 0\right)$ to list $\mathcal{L}_{2}$, sets $H \leftarrow H+1$ and returns $H$ to $\mathcal{A}_{2}$.

Challenge: $\mathcal{A}_{2}$ gives an identity $I D^{*}$ and two messages $M_{0}$ and $M_{1}$ to $\mathcal{B}$. $\mathcal{B}$ selects a random bit $\xi \in\{0,1\} . \mathcal{B}$ scans the list $\mathcal{L}_{2}$ to find $p k_{I D^{*}}=(Y, \pi)$ about $I D^{*}$ for the largest handle where $Y=e\left(g_{1}, v_{1}\right)^{\alpha \beta^{*}}$. $\mathcal{B}$ knows $\beta^{*}$ because the public key is not replaced. Otherwise, by using $g_{1}^{z} g_{2}^{v}, \mathcal{B}$ outputs the SF ciphertext $C^{*}=\left(C_{0}^{*}, \vec{C}^{*}, C_{1}^{*}, C_{2}^{*}\right)=\left(M_{\xi} \cdot e\left(v_{1}^{\beta^{*}}, g_{1}^{z} g_{2}^{v}\right)^{\alpha}\right.$, $\left.<\left(g_{1}^{z} g_{2}^{v}\right)^{x_{1}}, \cdots,\left(g_{1}^{z} g_{2}^{v}\right)^{x_{n}}>,\left(g_{1}^{z} g_{2}^{v}\right)^{d},\left(g_{1}^{z} g_{2}^{v}\right)^{a I D^{*}+b}\right)$.

From the SF ciphertext we can get SF parameters: $\vec{\delta}=<v x_{1}, \ldots, v x_{n}>, \delta_{1}=v d, \delta_{2}=v\left(a I D^{*}+b\right)$.

For the same reason as before, from the $\mathcal{A}_{2}$ 's point of view, $x_{1}, \ldots, x_{n}, d, a, b$ are random under modulo $p_{2}$ and the given ciphertext is properly distributed if $I D^{*}$ isn't the identity for the $(k+1)^{t h}$ handle.

For the $(k+1)^{t h}$ create inquiry, we can get the conclusion: If there is $G_{p_{2}}$ part in $T$, the decryption key towards the $(k+1)^{\text {th }}$ handle is semi-functional for the $C^{*}$. The NSF property is embodied as follows. $\vec{\theta} \cdot \vec{\delta}=\kappa v d \sum_{i=1}^{n} x_{i} w_{i} \bmod p_{2}, \theta_{1} \cdot \delta_{1}=-\kappa v d\left(a I D_{k+1}+b+\sum_{i=1}^{n} x_{i} w_{i}\right) \bmod p_{2}$, and $\theta_{2} \cdot \delta_{2}=-\kappa v d\left(a I D^{*}+b\right) \bmod p_{2}$.

So, if $T=g_{1}^{w} g_{2}^{\kappa} g_{3}^{\sigma}, \mathcal{B}$ imitates the $\mathcal{T}_{2}$ AltGame_ $k$ correctly when $I D^{*} \neq I D_{k+1} \bmod p_{2}$. Or else, $\mathcal{B}$ imitates $\mathcal{T}_{2}$ Game_k.

Furthermore, we should think over of NSF towards the $(k+1)^{\text {th }}$ handle in the following two cases.

(1) $I D^{*}=I D_{k+1} \bmod p_{2}, I D^{*} \neq I D_{k+1} \bmod N$.

(2) $I D^{*}=I D_{k+1} \bmod N$.

For the case (1), if $\mathcal{B}$ computes $a^{\prime}=\operatorname{gcd}\left(I D^{*}-I D_{k+1}, N\right)$ he can generate a nontrivial factor of $N$. Subgroup decision assumption is broken, which implies that assumption 1, 2, 3 are broken.

For the case (2), the challenge identity $I D^{*}$ is pointed out by the $(k+1)^{\text {th }}$ handle. In the case, $\mathcal{A}_{2}$ can't extract the decryption key of $I D^{*}$. In fact, $\mathcal{A}_{2}$ may obtain the certificate and some 
leakage of the decryption key of identity $I D^{*}$.

The following Lemma 14 is to prove that normal SF and NSF are indistinguishable.

Lemma 14. For the case (2) of Lemma 13, the advantage difference that $\mathcal{A}_{2}$ wins is negligible when the decryption key is changed from normal SF to NSF for the $(k+1)^{\text {th }}$ handle of identity $I D^{*}$ in the case of $\lambda_{d k}$ leakage of the decryption key, where $\lambda_{d k}=(n-2 c-1) \lambda$ and $c$ is a fixed positive constant.

Proof. If there is a PPT adversary $\mathcal{A}_{2}$ who may distinguish the SF and NSF decryption key/certificate with non-negligible advantage, we can construct a simulator $\mathcal{B}$ to break Lemma 10. That is to say, the advantage that $\mathcal{B}$ can succeed in distinguishing the distribution $\left(\vec{\delta}, f\left(\vec{\tau}^{\prime}\right)\right)$ and $(\vec{\delta}, f(\vec{\tau}))$ is non-negligible. Thus, there is a contradiction.

$\mathcal{B}$ runs Setup to give the $m p k$ and $m s k$ to $\mathcal{A}_{2}$. Because $\mathcal{B}$ has the $m s k$ and knows $g_{2} \in G_{p_{2}}$, he can generate normal and semi-functional keys. Thus, he can answer all the inquiries of $\mathcal{A}_{2}$.

Towards the $(k+1)^{t h}$ create inquiry about identity $I D, \mathcal{B}$ replies with the handle $H^{*}$ and doesn't create the keys. If $\mathcal{A}_{2}$ asks for the leakage of decryption key for $\left(H^{*}, f\right), \mathcal{B}$ encodes the leakage that $\mathcal{A}_{2}$ inquiries in Phase 1 for the $I D$ as a PPT function $f: Z_{p_{2}}^{n} \rightarrow 2^{\lambda_{d k}}$. This can be done if all the values of other keys and other variables of the challenge key are seen as fixed. Then $\mathcal{B}$ gets $(\vec{\delta}, f(\vec{\Gamma}))$, where $\vec{\Gamma}$ is $\vec{\tau}$ or $\vec{\tau}^{\prime}$ according to Lemma 10 . $\mathcal{B}$ returns $f(\vec{\Gamma})$ to $\mathcal{A}_{2}$ as the leakage about the $(k+1)^{\text {th }}$ handle, which defines the challenge keys in the following.

For the $(k+1)^{t h}$ create inquiry, $\mathcal{B}$ calculates normal certificate $\operatorname{Cert}_{I D}$ by using msk. $\mathcal{B}$ selects randomly $r_{1}, r_{2} \in Z_{p_{2}}$ and sets implicitly $G_{p_{2}}$ part in decryption key to be $g_{2}^{\vec{\Gamma}^{\prime}}$, where $\overrightarrow{\Gamma^{\prime}}$ is defined as $(\vec{\Gamma}, 0)+\left(0, \ldots, 0, r_{1}, r_{2}\right) . \mathcal{B}$ sets non- $G_{p_{2}}$ part in decryption key to satisfy the proper distribution. It is able to solve the case (2) of Lemma 13.

In certain circumstances, $\mathcal{A}_{2}$ gives the challenge messages. $\mathcal{B}$ selects $t_{2} \in Z_{p_{2}}$ which satisfies that $\delta_{n} r_{1}+t_{2} r_{2} \equiv 0 \bmod p_{2}$. By using $<\vec{\delta}, 0>+<0, \ldots, 0,0, t_{2}>$ as $G_{p_{2}}$ part where $\vec{\delta}$ has $n+1$ components, $\mathcal{B}$ further forms the challenge ciphertext. If $\vec{\delta}$ and $\vec{\Gamma}$ are orthogonal, the decryption key/certificate of the $(k+1)^{\text {th }}$ handle is NSF.

It is obvious that $\mathcal{B}$ is able to reply easily the inquiry in Phase 2 . Thus, by using the output of $\mathcal{A}_{2} . \mathcal{B}$ succeeds in distinguishing the distribution $\left(\vec{\delta}, f\left(\vec{\tau}^{\prime}\right)\right)$ and $(\vec{\delta}, f(\vec{\tau}))$ with non-negligible advantage.

If assumption 2 holds the $\mathcal{T}_{2}$ Game_ $k$ and $\mathcal{T}_{2}$ AltGame $k$ are indistinguishable from the adversary's point of view. We have $\left|A d v_{\mathcal{A}_{2}^{-}}^{\mathcal{T}_{2} \text { Game }_{-}{ }^{k}}\left(\lambda_{d k}\right)-A d v_{\mathcal{A}_{2}^{-}}^{\mathcal{T}_{2} \text { AltGame }{ }_{-}{ }^{k}}\left(\lambda_{d k}\right)\right| \leq \varepsilon$.

Lemma 15. If $\lambda_{d k}=(n-2 c-1) \lambda$ and assumption 2 holds, $\mathcal{T}_{2}$ A AltGame_k and $\mathcal{T}_{2}$ _Game_k+1 are indistinguishable from the adversary's point of view. That is $\left|A d v_{\mathcal{A}_{2}}^{\mathcal{T}_{2} \text { AltGame }{ }_{-}{ }^{2}}\left(\lambda_{d k}\right)-A d v_{\mathcal{A}_{2}}^{\mathcal{T}_{2} \text { Game }}{ }_{-}^{k+1}\left(\lambda_{d k}\right)\right| \leq \varepsilon$.

Proof. If there is a PPT adversary $\mathcal{A}_{2}$ who distinguishes the $\mathcal{T}_{2}{ }_{-}$AltGame_k and $\mathcal{T}_{2}$ _Game_k+1 with non-negligible advantage, we can construct a simulator $\mathcal{B}$ who breaks assumption 2. Firstly, $\mathcal{B}$ is given an instance $\left(N, G, G_{T}, e, g_{1}, g_{3}, g_{1}^{z} g_{2}^{v}, g_{2}^{u} g_{3}^{\rho}\right) . \mathcal{B}$ is given a challenge item $T$ which is $g_{1}^{\omega} g_{3}^{\sigma}$ or $g_{1}^{\omega} g_{2}^{\kappa} g_{3}^{\sigma}$. $\mathcal{B}$ interacts with $\mathcal{A}_{2}$ as follows.

Setup Phase: $\mathcal{B}$ firstly creates composite order bilinear groups $\left(N=p_{1} p_{2} p_{3}, G, G_{T}, e\right) . \mathcal{B}$ 
selects $a, b, d \in Z_{N}$ uniformly at random and sets $u_{1}=g_{1}^{a}, h_{1}=g_{1}^{b}$ and $v_{1}=g_{1}^{d}$. Given $g_{1} \in G_{p_{1}}$ and $g_{3} \in G_{p_{3}}, \mathcal{B}$ runs $G e n$ of $\Pi$ to generate the common reference string crs and selects $\operatorname{random}\left(\alpha, x_{1}, x_{2}, \ldots, x_{n}, r, y_{1}, y_{2}, \ldots, y_{n}\right) \in Z_{N}^{2 n+2}$ and a vector $\vec{\rho}=<\rho_{1}, \rho_{2}, \ldots, \rho_{n+3}>\in Z_{N}^{n+3}$ where $n \geq 2$ is an integer.

It outputs the master public keympk $=\left(N, G, G_{T}, e, e\left(g_{1}, v_{1}\right)^{\alpha}, g_{1}, g_{1}^{x_{1}}, \ldots, g_{1}^{x_{n}}, u_{1}, h_{1}, v_{1}, g_{3}, c r s\right)$ and the master secret key $m s k=\left(\vec{K}, K_{1}, K_{2}, K_{3}\right)=\left(<v_{1}^{y_{1}}, \ldots, v_{1}^{y_{n}}>, g_{1}^{\alpha} h_{1}^{-r} \prod_{i=1}^{n} g_{1}^{-x_{i} y_{i}}, v_{1}^{r}, u_{1}^{r}\right) * g_{3}^{\vec{\rho}}$.

Oracle Query: $\mathcal{B}$ may reply every query because he knows $\alpha, x_{i}, y_{i}, r$. Specifically, $\mathcal{B}$ answers the $j^{\text {th }}$ create query for identity $I D_{j}$ as follows.

$\mathcal{B}$ generates normal certificate $\operatorname{Cert}_{I D_{j}}=\left(\vec{D}, D_{1}, D_{2}\right)$ by using msk. The $\operatorname{Cert}_{I D_{j}}$ can leak information. $\mathcal{B}$ runs SetPrivateKey to get $\beta$ and runs SetPublicKey to get $p k_{I D_{j}}$.

(a) If $j \leq k, \mathcal{B}$ selects randomly vectors $\vec{\rho}_{1}, \vec{\gamma}_{1}, \vec{\rho}_{2}, \vec{\gamma}_{2} \in Z_{N}^{n+2}$ and calculates the decryption key $\widetilde{\operatorname{Cert}_{I D_{j}}}=\left(\vec{D}, D_{1}, D_{2}\right) *\left(g_{2}^{\mu} g_{3}^{\rho}\right)^{\overrightarrow{\gamma_{1}}} * g_{3}^{\vec{\rho}_{1}}, \widetilde{d k_{I D_{j}}}=\left(\vec{S}, S_{1}, S_{2}\right) *\left(g_{2}^{\mu} g_{3}^{\rho}\right)^{\overrightarrow{\gamma_{2}}} * g_{3}^{\vec{\rho}_{2}} \cdot \mathcal{B}$ adds $\left(H, I D_{j}\right.$, $\left.I D_{j}^{\prime}, p k_{I D_{j}}, s k_{I D_{j}}, \overline{\operatorname{Cert}_{I D_{j}}}, \widetilde{d k_{I D_{j}}}, 0\right)$ to the list $\mathcal{L}_{2}$.

(b) If $j>k+1, \mathcal{B}$ calculates normally the decryption key $d k_{I D_{j}}$ and adds $\left(H, I D_{j}, I D_{j}^{\prime}, p k_{I D_{j}}\right.$, $\left.s k_{I D_{j}}, \operatorname{Cert}_{I D_{j}}, d k_{I D_{j}}, 0\right)$ to the list $\mathcal{L}_{2}$.

(c) If $j=k+1, \mathcal{B}$ selects randomly vectors $\vec{\rho}_{1}, \vec{\rho}_{2}, \vec{\gamma}_{1} \in Z_{N}^{n+2}$ and $\left(z_{1}, z_{2}, \ldots, z_{n}\right) \in Z_{N}^{n}$. By using the challenge item $\mathcal{B}$ calculates the certificate $\overline{\operatorname{Cert}_{I D_{k+1}}}=\left(<T^{d_{1} z_{1}}, \ldots, T^{d_{1} z_{n}}\right\rangle, T^{-\left(a I D_{k+1}+b\right)} *$ $\left.\prod_{i=1}^{n} T^{-x_{i} z_{i}} * g^{\alpha}, T^{d}\right) * g_{3}^{\vec{\rho}_{1}}$.

$\mathcal{B}$ invokes SetDecryptKey to get decryption key $\left(\vec{S}, S_{1}, S_{2}\right)$ and calculates SF decryption key $\widetilde{d k_{I D_{j}}}=\left(\vec{S}, S_{1}, S_{2}\right) *\left(g_{2}^{\mu} g_{3}^{\rho}\right)^{\vec{\gamma}_{2}} * g_{3}^{\vec{\rho}_{2}}$. $\mathcal{B}$ adds $\left(H, I D_{j}, I D_{j}^{\prime}, p k_{I D_{j}}, s k_{I D_{j}}, \overline{\operatorname{Cert}_{I D_{j}}}, \widetilde{d k_{I D_{j}}}, 0\right)$ to the list $\mathcal{L}_{2}$.

If $T=g_{1}^{\omega} g_{3}^{\sigma}$, from the $\mathcal{B}$ 's point of view, the decryption key is properly distributed because no $G_{p_{2}}$ part is in $T$.

If $T=g_{1}^{\omega} g_{2}^{\kappa} g_{3}^{\sigma}$, the decryption key is semi-functional and the corresponding parameters are $\vec{\eta}=<\kappa d_{1} z_{1}, \ldots, \kappa d_{1} z_{n}>, \eta_{1}=-\kappa\left(a I D_{k+1}+b+\sum_{i=1}^{n} x_{i} z_{i}\right)$ and $\eta_{2}=\kappa d$.

Because $z_{1}, \ldots, z_{n}, x_{1}, \ldots, x_{n}, d, a, b$ are merely calculated by modulo $p_{1}$ in $m p k$, from the $\mathcal{A}_{2}$ 's point of view, they are random by modulo $p_{2}$. Thus, the SF decryption key is properly distributed.

$\mathcal{B}$ sets $H \leftarrow H+1$ and returns $H$ to $\mathcal{A} 2$.

Challenge: It is run in the same way as the Challenge phase of Lemma 13.

The NSF property is analyzed in a similar way as that of Lemma 13.

To summarize, if the assumption 2 holds and the amount of leakage adds up to $\lambda_{d k}=(n-2 c-1) \lambda$, $\mathcal{T}_{2}$ Game $k$ and $\mathcal{T}_{2}$ AltGame $k$ are indistinguishable from the adversary's point of view. Likewise, $\mathcal{T}_{2}{ }_{-}$AltGame_ $k$ and $\mathcal{T}_{2}$ Game_ $k+1$ are indistinguishable from the adversary's point of view. Thus, $\mathcal{T}_{2} \_$Game $k$ and $\mathcal{T}_{2}$ Game $e_{-}+1$ are indistinguishable from the adversary's point of view. We get $\left|A d v_{\mathcal{A}_{2}}^{\mathcal{T}_{2} \text { AltGame }{ }_{-}{ }^{-}}\left(\lambda_{d k}\right)-A d v_{\mathcal{A}_{2}^{-}}^{\mathcal{T}_{2} \text { Game }{ }_{-}{ }^{k+1}}\left(\lambda_{d k}\right)\right| \leq \varepsilon$. 
By Lemma 13 to Lemma 15, we have

$$
\begin{aligned}
& \left|A d v_{\mathcal{A}_{2}}^{\mathcal{T}_{2} \text { Game }{ }_{-}{ }^{k}}\left(\lambda_{d k}\right)-A d v_{\mathcal{A}_{2}}^{\mathcal{T}_{2} \text { Game }}{ }^{k+1}\left(\lambda_{d k}\right)\right|
\end{aligned}
$$

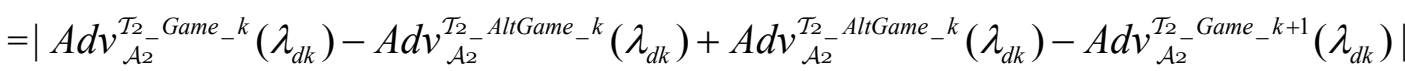

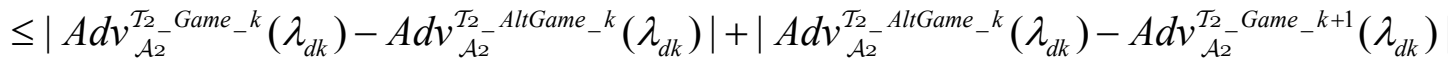

$$
\begin{aligned}
& =\varepsilon+\varepsilon=2 \varepsilon \text {. This finishes Lemma 12. Thus, we have } \\
& \left|A d v_{\mathcal{A}_{2}^{2}}^{\mathcal{T}_{2} \text { Game }{ }_{-}{ }^{k}}\left(\lambda_{d k}\right)-A d v_{\mathcal{A}_{2}^{-}}^{\mathcal{T}_{2} \text { Game }{ }_{-} k+1}\left(\lambda_{d k}\right)\right| \leq \varepsilon .
\end{aligned}
$$

Lemma 16. Under the assumption 3 , the advantage that PPT adversary succeeds in distinguishing $\mathcal{T}_{2}$ Game $Q$ and $\mathcal{T}_{2}$ Game_Final is negligible. That is $\mid A d v_{\mathcal{A}_{2}^{-}}^{\mathcal{T}_{2} \text { Game } Q_{-}}\left(\lambda_{d k}\right)-A d v_{\mathcal{A}_{2}}^{\mathcal{T}_{2} \text { Game }}$ Final $_{-}\left(\lambda_{d k}\right) \mid \leq \varepsilon$.

Proof. If there is a PPT adversary $\mathcal{A}_{2}$ who succeeds in distinguishing $\mathcal{T}_{2}$ _Game $Q$ and $\mathcal{T}_{2}$ _Game_Final with non-negligible advantage, we are able to construct an algorithm $\mathcal{B}$ to break the assumption $3 . \mathcal{B}$ is given an instance $\left(N, G, G_{T}, e, g_{1}, g_{2}, g_{3}, g_{1}^{\alpha} g_{2}^{v}, g_{1}^{s} g_{2}^{u}\right) . \mathcal{B}$ is given a challenge item $T$ which is $g_{1}^{\alpha s}$ or a random value in $G . \mathcal{B}$ interacts with $\mathcal{A}_{2}$ as follows.

Setup: $\mathcal{B}$ randomly selects $x_{1}, \ldots, x_{n}, a, b, d \in Z_{N}$ and sets $m p k=\left(e\left(g_{1}^{\alpha} g_{2}^{v}, v_{1}\right), g_{1}^{x_{i}}, u_{1}=g_{1}^{a}\right.$, $\left.h_{1}=g_{1}^{b}, v_{1}=g_{1}^{d}\right) . \mathcal{B}$ randomly selects the vectors $\vec{\rho}, \vec{\gamma} \in Z_{N}^{n+3}$ and $\left(y_{1}, \ldots, y_{n}, r\right) \in Z_{N}^{n+1}$ and then calculates the SF master secret key $m s k=\left(\left\langle v_{1}^{y_{1}}, \ldots, v_{1}^{y_{n}}\right\rangle, h_{1}^{-r} \prod_{i=1}^{n} g_{1}^{-x_{i} y_{i}}, v_{1}^{r}, u_{1}^{r}\right) * g_{2}^{\vec{\gamma}} * g_{3}^{\vec{\rho}}$.

Oracle Query: By using the SF master secret key $\mathcal{B}$ simulates all oracles.

Challenge: $\mathcal{A}_{2}$ gives an identity $I D^{*}$ and two messages $M_{0}^{*}$ and $M_{1}^{*}$ to $\mathcal{B}$. $\mathcal{B}$ selects a random bit $\xi \in\{0,1\} . \mathcal{B}$ scans the list $\mathcal{L}_{2}$ to find $p k_{I D^{*}}=(Y, \pi)$ about $I D^{*}$ for the largest handle where $Y=e\left(g_{1}, v_{1}\right)^{\alpha \beta^{*}}$. Because the $\mathcal{A} 2$ doesn't replace the public key $\mathcal{B}$ knows $\beta^{*}$. By using $g_{1}^{s} g_{2}^{u}$ and $T$ given from the assumption, $\mathcal{B}$ outputs the SF ciphertext $C^{*}=\left(C_{0}^{*}, \vec{C}^{*}, C_{1}^{*}, C_{2}^{*}\right)$ $=\left(M_{\xi}^{*} \cdot e\left(T, v_{1}^{\beta^{*}}\right),\left(g_{1}^{s} g_{2}^{\mu}\right)^{x_{i}},\left(g_{1}^{s} g_{2}^{\mu}\right)^{d},\left(g_{1}^{s} g_{2}^{\mu}\right)^{a I D^{*}+b}\right)$.

From the $\mathcal{A} 2$ 's point of view, $x_{1}, \ldots, x_{n}, d, a, b$ are random under modulo $p_{2}$. So, the given SF ciphertext is properly distributed.

If $T=g_{1}^{\alpha s}$, the ciphertext is distributed uniformly at random. In this case, $\mathcal{B}$ correctly simulates the $\mathcal{T}_{2}$ _Game_ $Q$. If $T$ is a random element of $G$, the item $C_{0}^{*}$ is random. Thus the ciphertext is $\mathrm{SF}$ for a random message. In this case, $\mathcal{B}$ correctly simulates the $\mathcal{T}_{2}$ _Game_Final. $\mathcal{B}$ breaks assumption 3 by using the output of $\mathcal{A}_{2}$. There is a contraction. We get

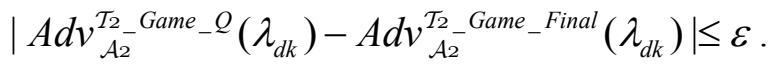

All in all, under the above seven Lemma 10 to Lemma 16 we know that $\mathcal{T}_{2}$ Game $R$ and $\mathcal{T}_{2}$ _Game_Final are indistinguishable from the adversary's point of view. What's more, $A d v_{\mathcal{A}_{2}}^{\mathcal{T}_{2} \text { Game }_{-} \text {Final }}\left(\lambda_{d k}\right) \leq \varepsilon$ is proved in Theorem 6.8 of the full version of [26]. To sum up, $A d v_{\mathcal{A}_{2}}^{\mathcal{T}_{2} \text { Game }{ }_{-}{ }^{R}}\left(\lambda_{d k}\right) \leq \varepsilon$. In addition, Lemma 10 proves the leakage bound. Thus, we complete the proof of Theorem 2. 